\title{
LARGE POPULATION STOCHASTIC DYNAMIC GAMES: CLOSED-LOOP MCKEAN-VLASOV SYSTEMS AND THE NASH CERTAINTY EQUIVALENCE PRINCIPLE*
}

\author{
MINYI HUANG ${ }^{\dagger}$, ROLAND P. MALHAMÉ $\ddagger$, AND PETER E. CAINES $\$$
}

\begin{abstract}
We consider stochastic dynamic games in large population conditions where multiclass agents are weakly coupled via their individual dynamics and costs. We approach this large population game problem by the so-called Nash Certainty Equivalence (NCE) Principle which leads to a decentralized control synthesis. The McKean-Vlasov NCE method presented in this paper has a close connection with the statistical physics of large particle systems: both identify a consistency relationship between the individual agent (or particle) at the microscopic level and the mass of individuals (or particles) at the macroscopic level. The overall game is decomposed into (i) an optimal control problem whose Hamilton-Jacobi-Bellman (HJB) equation determines the optimal control for each individual and which involves a measure corresponding to the mass effect, and (ii) a family of McKean-Vlasov (M-V) equations which also depend upon this measure. We designate the NCE Principle as the property that the resulting scheme is consistent (or soluble), i.e. the prescribed control laws produce sample paths which produce the mass effect measure. By construction, the overall closed-loop behaviour is such that each agent's behaviour is optimal with respect to all other agents in the game theoretic Nash sense.
\end{abstract}

Key words: Stochastic dynamic games, large populations, multi-class agents, interacting particle systems, statistical physics, decentralized control, Hamilton-Jacobi-Bellman equation, McKeanVlasov equation, Nash equilibria

1. Introduction. The modelling and analysis of dynamic systems with many interacting agents has gained research attention from a wide range of disciplines. In this paper, the investigation of large weakly coupled systems has its motivation coming from many complex phenomena arising in engineering and socioeconomic settings, among others, for instance, dynamic economic models involving competing agents $[18,14,35,25]$, biological models on animal competition and conflicts [36, 38], wireless power control [23, 24], road traffic engineering [46, 22], and shared data buffer modelling [3]. Also, large-scale weakly coupled stochastic dynamic systems appear in

\footnotetext{
* Dedicated to Professor Tyrone Duncan on the occasion of his 65th birthday. Work partially supported by ARC and NSERC. The authors gratefully acknowledge the references to fundamental work of McKean and Vlasov given them by Martin Clark and Sanjoy Mitter.

${ }^{\dagger}$ Minyi Huang is with the Department of Information Engineering, Research School of Information Sciences and Engineering, The Australian National University, Canberra, ACT 0200, Australia. Email: minyi.huang@rsise.anu.edu.au. Please address all correspondence to this author.

${ }^{\ddagger}$ Roland P. Malhamé is with the Department of Electrical Engineering, École Polytechnique de Montréal, Montreal, QC H3C 3A7, Canada, and also affiliated with GERAD, Montreal. E-mail: roland.malhame@polymtl.ca.

$\S$ Peter E. Caines is with the Department of Electrical and Computer Engineering, McGill University, Montreal, QC H3A 2A7, Canada. E-mail: peterc@cim.mcgill.ca.
} 
mathematical biology and have received justification by field observations [41]. Usually, a key common feature to these systems is that while each agent only receives a negligible influence from any other given individual, the effect of the overall population is significant to each agent.

In this paper, we study the optimization of large-scale nonlinear stochastic systems wherein many agents are each coupled with others via the individual dynamics and costs. This kind of weak coupling in both dynamics and costs is used to model the mutual impact of agents during competitive decision-making. In particular, the dynamic coupling specifies the impact of the environment on an individual's decisionmaking, and the underlying model takes the form of weakly coupled diffusions subject to individual controls. We aim to develop a methodology for multi-agent optimization with local information. In contrast to the extensive literature on dynamic games (see, e.g., $[6,42,16,31,45])$, we are particularly interested in large populations. We note that games with a large or infinite population have long been a major research area in game theory [33, 1, 2, 40, 43, 19], but traditionally most work has been based upon static models. And moreover, in our modelling each agent only has local information about its own state and dynamics in addition to the statistical property about the population parameter variation. This leads to a situation of decision with incomplete information [20, 21, 4, 39]. In general, for analysis of such games, Harsanyi's approach via Bayesian players [20] is important, though it involves great complexity in specifying the types of different players. However, in large population conditions, it is possible to develop efficient decision-making without paying excessive attention to the fine details of the system structure since each individual agent's effect is extremely weak with respect to the overall population's behaviour which becomes more relevant to a given agent's optimization as the population increases. Such an intuitive fact will provide the main motivation in developing our methodology which has a close connection with large particle systems and statistical physics [5, 44, 34, 32, 11].

Based upon the interaction between the individual and mass, we develop state aggregation for the underlying dynamic models which extracts the overall effect a given agent receives from the population. Our general methodology based upon state aggregation and upon individual-mass interaction was first developed for a population with linear individual dynamics or mild nonlinearity [23, 25, 28, 29, 27, 26, 30], combined with quadratic individual costs, which usually led to explicit calculations for the individual strategies. The aim of this paper is to extend this general approach to fully nonlinear models. Our analysis is facilitated by considering a controlled McKeanVlasov (M-V) equation associated with the large population limit. The key step is to construct a mutually consistent pair of (i) the mass effect and (ii) the individual strategies such that the latter not only each constitute an optimal response to the mass 
effect but also collectively produce that mass effect. By the NCE Methodology we mean the overall game decomposition into a (non-standard) optimal control problem whose HJB equation involves a mass effect measure and a closed-loop McKean-Vlasov equation; these two parts are related to each other by the optimal control law derived from the former. In this setting, we designate the NCE Principle as the property that the resulting scheme is consistent in the sense that the prescribed control laws produce sample paths which produce the mass effect with respect to which the optimal control is derived via the HJB equation. It is a property of this overall closed-loop behaviour that each agent's optimal behaviour with respect to all other agents holds in the game theoretic Nash sense.

So it turns out that the application of the NCE methodology amounts to determining a certain mass effect such that in the first step we solve a stochastic optimal control problem which generates the individual strategies, and such that in the second step, the closed-loop M-V equation will generate the same mass effect which has been used in the first place. In carrying out these two steps, we introduce the so-called best response map and measure-flow inducing map. The solution to the overall problem, and hence the demonstration of the NCE Principle, or Property, relies on finding a fixed point to the composite action of the two maps in the appropriate metrized space of measures. The fixed point analysis is facilitated by introducing the so-called Vasershtein metric for probability measures on the space of continuous functions [13, 44].

The rest of the paper is organized as follows. In Section 2 we introduce the stochastic dynamic game model involving a set of interacting diffusions, and the wellknown McKean-Vlasov equation for interacting particles. In Section 3 we describe the NCE principle which yields the HJB equation together with individual strategies, and the closed-loop M-V equation. Section 4 introduces some important assumptions for the system dynamics. Sections 5 and 6 analyze the decoupled HJB equation and M-V equation, respectively. In Section 7 we present an existence result for the consistent pair under suitable regularity and gain conditions. Section 8 is devoted to an asymptotic equilibrium analysis.

For the sake of exposition, we make a few conventions about notation. For a system involving many scalar state components (e.g., $z_{i}, y_{i}$, etc.), we use the integervalued subscript as the label for a certain agent. When the system involves only a single agent or particle (e.g., $x_{t}, w_{t}$ ), we may use the real-valued subscript to indicate time. Throughout the paper, $|\cdot|$ denotes the Euclidean norm of a vector. The integer $n$ is reserved to denote the population size of the game. We use $C, C_{1}, C_{2}$, etc. to denote a generic constant independent of the population size, and they may vary from place to place. 
2. The Weakly Coupled Systems. We first formulate the large population stochastic dynamic game, which is then followed by the introduction of the interacting particle (IP) system. The motivation for such an organization is that, while the former will be the focus of our analysis in this paper, the modelling and optimization approach in the nonlinear stochastic dynamic game problem has a close connection with the modelling approach in the latter, especially with respect to the individualmass interaction aspect and the microscopic analysis based upon single agents or particles.

2.1. The Stochastic Dynamic Game. We consider an $n$ dimensional nonlinear stochastic system where the evolution of each state component is described by

$$
d z_{i}=(1 / n) \sum_{j=1}^{n} f_{a_{i}}\left(z_{i}, u_{i}, z_{j}\right) d t+\sigma d w_{i}, \quad 1 \leq i \leq n, \quad t \geq 0
$$

where $\left\{w_{i}, 1 \leq i \leq n\right\}$ denotes $n$ independent standard scalar Wiener processes. The initial states $z_{i}(0)$ are mutually independent, and also independent of $\left\{w_{i}, 1 \leq i \leq n\right\}$. In addition, $E\left|z_{i}(0)\right|^{2}<\infty$. Each state component shall be referred to as the state of the corresponding agent (also to be called player). The control input $u_{i}$ takes its values in a compact set $U=[\alpha, \beta]$. The function $f_{a_{i}}$ is from $\mathbb{R} \times U \times \mathbb{R}$ to $\mathbb{R}$. For simplicity of analysis we take the diffusion coefficient to be the same constant $\sigma>0$ for the $n$ agents. Unless otherwise stated, throughout the paper $z_{i}$ is described by the dynamics (1).

The nonlinear functions $f_{(\cdot)}$ are indexed by $a \in \mathcal{A}$ where $a$ is called the dynamic parameter and $\mathcal{A}$ is an index set. Note that we indicate no explicit dependence of $f_{(\cdot)}$ on $a$, and for different values of $a, f_{a}$ is allowed to take different forms. For the $i$ th agent, its dynamic parameter takes the specific value $a_{i} \in \mathcal{A}$. The dynamic parameter may vary from agent to agent; this property is used to describe the heterogeneity of the population. In the special case when $\mathcal{A}$ is a singleton, we get a population of uniform agents.

In the analysis below we assume a finite set $\mathcal{A}=\left\{\theta_{1}, \cdots, \theta_{K}\right\}$ for modelling multi-class agents, where $K$ is the number of agent classes. We further assume that the distribution for the initial state depends upon the class of the agent. Hence we may denote $\mu_{0}^{a_{i}}$ for the initial distribution of $z_{i}$, and there are a total of $K$ classes of initial distributions, listed by $\mu_{0}^{\theta_{1}}, \cdots, \mu_{0}^{\theta_{K}}$.

The cost function for the $i$ th agent is given in the form:

$$
J_{i}\left(u_{i}\right) \triangleq E \int_{0}^{T}\left[(1 / n) \sum_{j=1}^{n} L\left(z_{i}, u_{i}, z_{j}\right)\right] d t,
$$


where $T \in(0, \infty)$ is the terminal time, $L$ is a nonlinear function from $\mathbb{R} \times U \times \mathbb{R}$ to $\mathbb{R}_{+}=[0, \infty)$.

To emphasize the control objective in relation to its own state and control processes, it is possible to consider a more general form for the cost function of the $i$ th agent:

$$
J_{i}^{\prime}\left(u_{i}\right) \triangleq E \int_{0}^{T} \Theta\left(z_{i}, u_{i},(1 / n) \sum_{j=1}^{n} L\left(z_{i}, u_{i}, z_{j}\right)\right) d t,
$$

where $\Theta$ is a function from $\mathbb{R} \times U \times \mathbb{R}$ to $\mathbb{R}_{+}$. However, in this paper we will only consider the optimization based upon the cost (2), and under suitable conditions, our analysis may be easily adapted to deal with the cost structure (3).

For the system configuration $z=\left(z_{1}, \cdots, z_{n}\right)$, define the empirical distribution $\varepsilon_{z}=(1 / n) \sum_{i=1}^{n} \delta_{z_{i}}$ where $\delta$ • is the Dirac measure. Then the coupling terms in the individual dynamics and costs are functionals of $\varepsilon_{z}$ which is insensitive to the ordering of the entries in $z$. This feature is important and will be exploited in developing the aggregation technique for control synthesis.

For the above system, the objective is to seek individual control strategies and appropriately characterize their optimality, and a standard approach is to analyze Nash (equilibrium) strategies. However, this approach requires that each agent has full information on the states and dynamic parameters of all agents, which results in very high control complexity under large-population conditions. This motivates us to search for lower complexity control strategies.

In specifying the structure of the individual dynamics and costs, we assume that neither $f_{a_{i}}$ nor $L$ explicitly depend upon the control of other agents. However, each $z_{i}$ is under an indirect influence of $u_{j}, j \neq i$ via the coupling state variable $z_{j}$ appearing in $f_{a_{i}}\left(z_{i}, u_{i}, z_{j}\right)$. For illustrating this class of models, we consider a concrete example as follows.

EXAMPLE 1. We take $f_{a_{i}}\left(z_{i}, u_{i}, z_{j}\right)=f_{a_{i}}^{0}\left(z_{i}, u_{i}\right)+f_{a_{i}}^{1}\left(z_{j}\right)$ and $L\left(z_{i}, u_{i}, z_{j}\right)=$ $L^{0}\left(z_{i}, u_{i}\right)+L^{1}\left(z_{j}\right)$. This gives the drift term $(1 / n) \sum_{j=1}^{n} f_{a_{i}}\left(z_{i}, u_{i}, z_{j}\right)=f_{a_{i}}^{0}\left(z_{i}, u_{i}\right)+$ $(1 / n) \sum_{j=1}^{n} f_{a_{i}}^{1}\left(z_{j}\right)$ and cost integrand $(1 / n) \sum_{j=1}^{n} L\left(z_{i}, u_{i}, z_{j}\right)=L^{0}\left(z_{i}, u_{i}\right)+(1 / n)$ $\sum_{j=1}^{n} L^{1}\left(z_{j}\right)$, corresponding to (1) and (2), respectively.

The modelling in Example 1 yields a typical situation where each agent's dynamics and cost are closely related to its own state and control selection while receiving an average impact from the population. This reflects an important feature in many practical situations for decision-making.

2.2. The Interacting Particle System. In an interacting particle (IP) system, the state evolution of an individual particle is affected by an empirical average of coupling terms with all other particles. Mathematically, this leads to a set of weakly 
coupled diffusions, each describing the motion of a single particle, where an averaging across the population produces the coupling term in the individual dynamics. We introduce the following dynamics [12] in the form of $N$ coupled stochastic differential equations (SDE):

$$
d x_{i}=(1 / N) \sum_{k=1}^{N} b\left(x_{i}, x_{k}\right) d t+\sigma d w_{i}, \quad 1 \leq i \leq N, \quad t \geq 0,
$$

where $b(\cdot, \cdot)$ is a function from $\mathbb{R}^{2}$ to $\mathbb{R}, N$ is the number of particles and all $x_{i}$ 's are assumed to have i.i.d. initial conditions at $t=0$. Here we assume $x_{i}$ is a scalar although the modelling is also applicable to the case of vector particle states. The noises $\left\{w_{i}, 1 \leq i \leq N\right\}$ are $N$ independent Wiener processes independent of the initial conditions $x_{i}(0), 1 \leq i \leq N$. Let $\varepsilon_{x}=(1 / N) \sum_{i=1}^{N} \delta_{x_{i}}$ denote the empirical measure of the particle configuration $\left(x_{1}, x_{2}, \cdots, x_{N}\right)$. Then the drift term in (4) may be expressed as a function of $x_{i}$ and $\varepsilon_{x}$.

For this class of particle models, one can achieve a remarkable degree of economy in the description of population dynamics, by expressing the aggregate coupling term in terms of an expectation over a typical individual's probability distribution function which evolves with time. This is based upon the following intuition: as the number of particles grows to infinity, the particles become essentially indistinguishable while each individual being negligible, and furthermore, there is a decoupling effect such that a single particle's statistical properties can effectively approximate the empirical distribution produced by all particles [10]. More specifically, as $N$ tends to infinity, the individual dynamics may be written in the limiting form:

$$
d x_{t}=b\left[x_{t}, \mu_{t}\right] d t+\sigma d w_{t}, \quad t \geq 0
$$

which is the celebrated McKean-Vlasov (M-V) equation. Here $b\left[x, \mu_{t}\right]=\int b(x, y) \mu_{t}$ $(d y)$ for some probability distribution $\mu_{t}$ on $\mathbb{R}$. This equation, as well as its variants, has been extensively studied in physics, stochastic analysis, and partial differential equations $[12,44,37,7,8]$. The noise $w_{t}$ may be determined in different ways. For instance, if one intends to approximate $x_{1}$ in (4) by $x_{t}$, one may set $w_{t}=w_{1}$ as the driving Brownian motion in (5) and $x_{0}=x_{1}(0)$. Note that by introducing the density function $p_{t}(x)$, associated with $\mu_{t}$ for $x_{t}$, one may recast (5) in the form of a Fokker-Planck equation whose coefficients depend upon the density $p_{t}(x)$ itself.

Definition 2. A pair $\left(x_{t}, \mu_{t}\right), t \geq 0$, is said to be a consistent pair if $x_{t}$ is a solution to the $S D E(5)$ and $\mu_{t}$ is its distribution at time $t$, i.e., $P\left(x_{t} \leq \alpha\right)=$ $\int_{-\infty}^{\alpha} \mu_{t}(d y)$ for all $\alpha \in \mathbb{R}$ and $t \geq 0$.

It is obvious that $\mu_{0}$ in Definition 2 is determined as the distribution of $x_{0}$. For a detailed analysis on the existence and uniqueness of a solution to (5) and asymptotic 
properties, see $[7,8]$. For a weak convergence relationship between solutions of (4) and (5), and large deviation analysis for $\mu_{t}$, see [44, 12] and references therein.

3. The Nash Certainty Equivalence Principle via Population Limit. For the above $n$-agent dynamic game, as $n \rightarrow \infty$, we will use the M-V equation (5), as well as the notion of a consistent state-distribution pair, as a motivating scheme to develop reduction methods for the mass coupling term in the individual dynamics (1). Specifically, we attempt to use a probability distribution $\mu_{t}^{i}$ on $\mathbb{R}$, to approximate the empirical distribution of the sub-configuration $\left(z_{k_{1}}, \cdots z_{k_{l}}\right)$ of $\left(z_{1}, \cdots, z_{n}\right)$ at time $t$, which corresponds to the $l$ states sharing a dynamic parameter $a=\theta_{i}$. Roughly speaking, the parameter $a$ is used as a classifier for a family of empirical distributions each being induced by the same class of agents. The rationale for using the $K$ distributions $\mu_{t}^{i}, 1 \leq i \leq K$, to approximate the overall population effect is that as $n \rightarrow \infty$, if the population is well randomized so as to give a sufficient number of agents in each class appearing in the game, then it is possible to approximate the mass effect by the superposition of these $K$ distributions, provided that all agents have sufficiently weak coupling and generate their effect on any given agent additively in a certain manner. However, it should be clear that this is only a heuristic argument, and its justification via the exact characterization of the individual-mass interaction needs to be based upon rigorous mathematical analysis, which will be the main focus of what follows.

For the sequence $\left\{a_{i}, i \geq 1\right\}$ where $a_{i} \in \mathcal{A}$, we define the empirical distribution associated with the first $n$ agents

$$
F_{n}\left(\left\{\theta_{k}\right\}\right)=(1 / n) \sum_{i=1}^{n} 1_{\left(a_{i}=\theta_{k}\right)},
$$

which gives a discrete distribution function on $\mathcal{A}$ for each given $n$. We introduce the assumption below as a characterization of the population statistics.

(H0) There exists a distribution function $F$ on $\mathcal{A}=\left\{\theta_{1}, \cdots, \theta_{K}\right\}$, denoted as $\pi=\left(\pi_{1}, \cdots, \pi_{K}\right)$ (i.e., $\left.F\left(\left\{\theta_{k}\right\}\right)=\pi_{k}\right)$, such that $\lim _{n \rightarrow \infty} F_{n}\left(\left\{\theta_{k}\right\}\right)=F\left(\left\{\theta_{k}\right\}\right)$ for each $\theta_{k} \in \mathcal{A}$.

Before tackling the large population game, we first consider its limiting form via specifying the behaviour of a single agent, as in the M-V equation. To facilitate the exposition, we call a $K$-tuple $\left(\mu_{t}^{1}, \cdots, \mu_{t}^{K}\right)$ of $K$ probability measures on $\mathbb{R}$, defined for all $t \in[0, T]$, a probability measure flow on $[0, T]$. Also, we may simply call it a measure flow.

We write the dynamics of a representative agent with the scalar state variable $x_{t}$ :

$$
d x_{t}=f_{a}\left[x_{t}, u_{t}, \mu_{t}^{1}, \cdots, \mu_{t}^{K}\right] d t+\sigma d w_{t},
$$

where the distribution of $x_{0}$ will be specified, depending upon the value of $a$, as the common initial distribution of that class of agents, and the drift coefficient is defined 
as

$$
f_{a}\left[x, u, \mu_{t}^{1}, \cdots, \mu_{t}^{K}\right]=\sum_{i=1}^{K} \pi_{i} \int_{\mathbb{R}} f_{a}(x, u, y) \mu_{t}^{i}(d y),
$$

which results from the superposition of the average effects, each determined by integrating with respect to a certain $\mu_{t}^{i}$, of multi-class agents. We note that the state variable $x_{t}$, control variable $u_{t}$ and noise $w_{t}$ are associated with a specific value of $a$; however, for notational brevity, we do not include a matching index $a$ for $x_{t}, u_{t}$ and $w_{t}$. In fact, if we write equation (6) for $K$ agents in distinct classes, we will get a system of $K$ equations which have their own independent initial conditions and Brownian motions and are coupled by $\mu_{t}^{i}, 1 \leq i \leq n$. These equations are mutually independent if each individual control is adapted to the $\sigma$-algebra generated by the Brownian motion in the same equation.

And corresponding to (2), we define the cost function:

$$
J\left(u, \mu^{1}, \cdots, \mu^{K}\right) \triangleq E \int_{0}^{T} L\left[x_{t}, u_{t}, \mu_{t}^{1}, \cdots, \mu_{t}^{K}\right] d t,
$$

where

$$
L\left[x, u, \mu_{t}^{1}, \cdots, \mu_{t}^{K}\right]=\sum_{i=1}^{K} \pi_{i} \int_{\mathbb{R}} L(x, u, y) \mu_{t}^{i}(d y) .
$$

Within the context of the limiting game problem, we give the interpretation for the controlled system dynamics (6) as follows. The control $u_{t}$ should be sought such that (i) it is optimal for the minimization of $J\left(u, \mu^{1}, \cdots, \mu^{K}\right)$ when the measure flow $\left(\mu_{t}^{1}, \cdots, \mu_{t}^{K}\right), 0 \leq t \leq T$, is treated as an exogenous signal, and (ii) the distribution of $x_{t}$ in the closed-loop system coincides with $\mu_{t}^{i}$, i.e., $P\left(x_{t} \leq \alpha\right)=\int_{-\infty}^{\alpha} \mu_{t}^{i}(d y)$ for all $\alpha \in \mathbb{R}$ and $t \geq 0$, when the dynamic parameter is set as $a=\theta_{i}$. This forms the basis for our subsequent control synthesis and this control design scheme is called the Nash Certainty Equivalence (NCE) Methodology. Equation (6) may be looked at as a controlled McKean-Vlasov equation for multi-class agents with the control performance measured by (8). Notice that in step (i), $u_{t}$ is formally regarded as not affecting $\left(\mu_{t}^{1}, \cdots, \mu_{t}^{K}\right)$ during the strategy selection. The reason for so doing is that in relation to the game with a large but finite population, $\mu_{t}^{i}$ is used to model the collective effect of the $i$ th class of agents whose states are under their own controls and as such, it is expected to become asymptotically insensitive to the control of the individual agent in question. This consequently leads to a decoupled stochastic control problem involving an isolated agent, which is indicated by its dynamic parameter to be in one of the $K$ classes of agents. Subsequently, in the closed-loop equation we get $\mu_{t}^{i}$ as the distribution of $x_{t}$ associated with the parameter $a=\theta_{i}$. 
3.1. The NCE Methodology and the NCE Equation. Since the measure flow $\left(\mu_{t}^{1}, \cdots, \mu_{t}^{K}\right), 0 \leq t \leq T$, is treated as an exogenous signal in the NCE methodology, the control for the agent described by (6) and (8) is determined by a standard optimal control problem. We formally proceed to write the following equations for which the existence analysis will be developed subsequently. Assuming the measure flow $\left(\mu_{t}^{1}, \cdots, \mu_{t}^{K}\right), 0 \leq t \leq T$, is given first, we use $V_{a}(t, x),(t, x) \in$ $[0, T] \times \mathbb{R}$ to denote the value function of agents with parameter $a$, i.e., $V_{a}(t, x)=$ $\inf _{u(\cdot) \in \mathcal{U}} \int_{t}^{T} L\left[x_{s}, u_{s}, \mu_{s}^{1}, \cdots, \mu_{s}^{K}\right] d s$ subject to the dynamics (6). The admissible control set $\mathcal{U}$ consists of all controls such that $u_{t} \in U$ and is adapted to the $\sigma$-algebra $\sigma\left(x_{0}, w_{s}, s \leq t\right)$ with the associated Brownian $w_{t}$ in this agent's dynamics. We write the HJB equation

$$
-\frac{\partial V_{a}}{\partial t}=\inf _{u \in U}\left\{f_{a}\left[x, u, \mu_{t}^{1}, \cdots, \mu_{t}^{K}\right] \frac{\partial V_{a}}{\partial x}+L\left[x, u, \mu_{t}^{1}, \cdots, \mu_{t}^{K}\right]\right\}+\frac{\sigma^{2}}{2} \frac{\partial^{2} V_{a}}{\partial x^{2}},
$$

where the initial time-state pair is $(t, x) \in[0, T) \times \mathbb{R}$ and the terminal condition is $V_{a}(T, x)=0$.

We denote the resulting optimal control law by

$$
u_{t}=\varphi_{a}\left(t, x \mid \mu_{.}^{1}, \cdots, \mu_{.}^{K}\right),
$$

where $(t, x) \in[0, T] \times \mathbb{R}$. It should be noted that the notation in (10) indicates that the value of $\varphi_{a}$ at time $t$ depends upon the measure flow $\left(\mu_{t}^{1}, \cdots, \mu_{t}^{K}\right)$ on the whole interval $[0, T]$.

Substituting (10) into (6), we write the closed-loop dynamics

$$
d x_{t}=f_{a}\left[x_{t}, \varphi_{a}\left(t, x \mid \mu_{.}^{1}, \cdots, \mu_{.}^{K}\right), \mu_{t}^{1}, \cdots, \mu_{t}^{K}\right] d t+\sigma d w_{t}, \quad 0 \leq t \leq T .
$$

Now, the NCE methodology amounts to finding a solution $\left(x_{t}, u_{t}\right)$ for each of the $K$ classes of agents and a measure flow $\left(\mu_{t}^{1}, \ldots, \mu_{t}^{K}\right)$ such that (9)-(11) hold, where the distribution of $x_{t}$ in equation (11) is equal to $\mu_{t}^{i}$ for $t \in[0, T]$ when $a=\theta_{i} \in \mathcal{A}$.

Within the framework of the NCE methodology it is required that $\varphi_{a}$ is derived from the HJB equation, and in this setting equation (11) may be regarded as a generalized McKean-Vlasov equation where the right hand side has a functional dependence on the distributions of multi-class particles.

For a better appreciation of the interaction between the individual and the mass, we generate $K$ copies of (11) by taking $K$ distinct values of $a$ combined with different initial conditions and driving Brownian motions. This leads to the following coupled $\mathrm{M}-\mathrm{V}$ equation system:

$$
\left\{\begin{aligned}
d x_{1} & =f_{1}\left[x_{1}, \varphi_{1}\left(t, x_{1} \mid \mu_{.}^{1}, \cdots, \mu_{.}^{K}\right), \mu_{t}^{1}, \cdots, \mu_{t}^{K}\right] d t+\sigma d w_{1} \\
& \vdots \\
d x_{K} & =f_{K}\left[x_{K}, \varphi_{K}\left(t, x_{K} \mid \mu_{.}^{1}, \cdots, \mu_{.}^{K}\right), \mu_{t}^{1}, \cdots, \mu_{t}^{K}\right] d t+\sigma d w_{K}
\end{aligned}\right.
$$


where we write $f_{a}\left(\varphi_{a}\right.$, resp. $)$ as $f_{i}\left(\varphi_{i}\right.$, resp.) when $a=\theta_{i}$. The $K$ components have independent initial conditions and $\left.\mu_{t}^{k}\right|_{t=0}=\mu_{0}^{\theta_{k}}, 1 \leq k \leq K$, where $\left(\mu_{0}^{\theta_{1}}, \cdots, \mu_{0}^{\theta_{K}}\right)$ has been specified in Section 2.1. The independent Brownian motions here may be different from those in (1). The subsequent analysis is concerned with the existence of a consistent pair $\left(x_{1}, \cdots, x_{K}\right)$ and $\left(\mu^{1}, \cdots, \mu^{K}\right)$ solving the equation system (12) coupled by the measure flow where $\varphi_{i}$ is derived from the associated HJB equation with a corresponding parameter $a=\theta_{i}$.

4. Main Assumptions and Restriction on Measure Flows. Our strategy to approach the limiting game problem is to first detach the HJB equation (9) from the closed-loop M-V equation (11). In the analysis below, the measure flows such as $\left(\mu_{t}^{1}, \cdots, \mu_{t}^{K}\right), 0 \leq t \leq T$, do not necessarily satisfy (9)-(11) simultaneously.

We introduce the following assumptions:

(H1) $U$ is a compact interval.

(H2) For each $a \in \mathcal{A}, f_{a}(x, u, y)$ and $L(x, u, y)$ are continuous and bounded on $\mathbb{R} \times U \times \mathbb{R}$, and Lipschitz continuous in $(x, y)$, i.e., there exist constants $B_{i}>0$ such that

$$
\begin{aligned}
& \left|f_{a}(x, u, y)-f_{a}\left(x^{\prime}, u, y^{\prime}\right)\right| \leq B_{1}\left|x-x^{\prime}\right|+B_{2}\left|y-y^{\prime}\right|, \\
& \left|L(x, u, y)-L\left(x^{\prime}, u, y^{\prime}\right)\right| \leq B_{3}\left|x-x^{\prime}\right|+B_{4}\left|y-y^{\prime}\right|,
\end{aligned}
$$

for all $u \in U$ and $x, x^{\prime}, y, y^{\prime} \in \mathbb{R}$. In addition, there exists a modulus of continuity $m(\cdot):(0, \infty) \rightarrow \mathbb{R}_{+}$(satisfying $\left.m(0+)=0\right)$ such that $\sup _{x, y, u \neq u^{\prime}} \mid \psi(x, u, y)-$ $\psi\left(x, u^{\prime}, y\right) \mid \leq m\left(\left|u-u^{\prime}\right|\right)$, where $\psi$ stands for $f_{a}$ or $L$.

(H3) For both $f_{a}(x, u, y)$ and $L(x, u, y)$, their first and second order derivatives (w.r.t. $x$ ) are all uniformly continuous and bounded on $\mathbb{R} \times U \times \mathbb{R}$, and Lipschitz continuous in $y$.

(H4) For each $a \in \mathcal{A}, f_{a}(x, u, y)$ is Lipschitz continuous in $u \in U$, i.e., there exists $B_{5}>0$ such that $\left|f_{a}(x, u, y)-f_{a}\left(x, u^{\prime}, y\right)\right| \leq K_{5}\left|u-u^{\prime}\right|$ for any $u, u^{\prime} \in U$.

(H5) For any $q \in \mathbb{R}, a \in \mathcal{A}$ and any probability distribution $\nu(d y)$ on $\mathbb{R}$, the set

$$
S_{a}(x, q)=\arg \min _{u \in U}\left\{\int_{y \in \mathbb{R}}\left[q f_{a}(x, u, y)+L(x, u, y)\right] \nu(d y)\right\}
$$

is a singleton and the resulting $u$, as a function of $(x, q)$, is Lipschitz continuous in $(x, q) \in \mathbb{R}^{2}$, uniformly with respect to the choice of $\nu(d y)$.

The assumptions (H1)-(H2) are mostly standard conditions used in the stochastic control literature. In (H3), we only need the differentiability condition with respect to $x$ rather than $(x, y)$ since in the limiting game the $y$ component will be averaged out to lead to time-varying dynamics involving $(t, x)$.

We need (H4) and (H5) to ensure some regularity of the closed-loop McKeanVlasov equation in order to analyze its solvability. It should be noted that when (H4) 
holds, the modulus of continuity condition for $f_{a}$ in (H2) is automatically satisfied. The first part of (H5) may be satisfied under suitable convexity conditions with respect to $u$. We may give a simple example where $L$ contains a quadratic term $u^{2}$ multiplied by a positive term $g(x)$ and where $f_{a}$ is affine in $u$. For illustration, consider the model $f_{a}=f_{a}^{0}(x, y)+f_{a}^{1}(x, y) u$ and $L=L^{0}(x, y)+g(x, y) u^{2}$, where $\inf _{x, y} f_{a}^{1}(x, y)>0$ and $\inf _{x, y} g(x, y)>0$; by applying (H5) to such a pair $\left(f_{a}, L\right)$ for given $(x, q)$ and $\nu(d y)$, it leads to the minimization of a quadratic function in terms of the argument $u$ restricted to a compaction interval. Obviously this procedure yields a unique minimizer.

If the probability measure flow $\left(\mu_{t}^{1}, \cdots, \mu_{t}^{K}\right), 0 \leq t \leq T$, is fixed, $f_{a}\left[x, u, \mu_{t}^{1}, \cdots\right.$, $\left.\mu_{t}^{K}\right]$ and $L\left[x, u, \mu_{t}^{1}, \cdots, \mu_{t}^{K}\right]$ each become a function of $(t, x, u)$, and accordingly, we denote

$$
f_{a}^{*}(t, x, u) \triangleq f_{a}\left[x, u, \mu_{t}^{1}, \cdots, \mu_{t}^{K}\right], \quad L^{*}(t, x, u) \triangleq L\left[x, u, \mu_{t}^{1}, \cdots, \mu_{t}^{K}\right]
$$

In order to analyze the HJB equation involving $f^{*}$ and $L^{*}$, we need to impose some restrictions on the measure flow, which will be useful for examining the individual equations in (9)-(11). We introduce the following class $\mathcal{M}_{[0, T]}$ of measure flows.

Definition 3. A probability measure flow $\left(\mu_{t}^{1}, \cdots, \mu_{t}^{K}\right)$ on $[0, T]$ is in $\mathcal{M}_{[0, T]}$, if there exists $\beta \in(0,1]$ such that for any bounded and Lipschitz continuous function $\psi$ on $\mathbb{R}$,

$$
\sup _{1 \leq j \leq K}\left|\int_{\mathbb{R}} \psi(y) \mu_{t^{\prime}}^{j}(d y)-\int_{\mathbb{R}} \psi(y) \mu_{t^{\prime \prime}}^{j}(d y)\right| \leq B_{6}\left|t^{\prime}-t^{\prime \prime}\right|^{\beta}
$$

for all $t^{\prime}, t^{\prime \prime} \in[0, T]$, where for the given $\left(\mu_{t}^{1}, \cdots, \mu_{t}^{K}\right)$, the constant $B_{6}$ may be selected to depend only upon the Lipschitz constant of $\psi$. The constant $\beta$, to be called the Hölder exponent, depends upon the specific $\left(\mu_{t}^{1}, \cdots, \mu_{t}^{K}\right)$.

The set $\mathcal{M}_{[0, T]}$ is nonempty since we may take all $\mu_{t}^{j}, 1 \leq j \leq K$ and $0 \leq t \leq T$, to be the Dirac measure at any constant $y_{0}$. We give some explanation on (14) by relating it to weak convergence of measures. Let $t^{\prime}$ be fixed and take $t^{\prime \prime} \rightarrow t^{\prime}$. If $u_{t^{\prime \prime}}$ is only known to weakly converge to $u_{t^{\prime}}$, the left hand side is a vanishing term for any bounded and continuous function $\psi$, but this in general leads to no explicit vanishing rate. Thus in defining $\mathcal{M}_{[0, T]}$, the convergence rate is strengthened.

Proposition 4. Let $f_{a}^{*}, a \in \mathcal{A}$, and $L^{*}$ be defined by (13) for which $\left(\mu_{t}^{1}, \cdots, \mu_{t}^{K}\right)$ $\in \mathcal{M}_{[0, T]}$ is fixed with Hölder exponent $\beta$ in (14), and in the following we assume (H1) always holds. We have:

(i) Under (H2), $f_{a}^{*}$ and $L^{*}$ are continuous and bounded on $[0, T] \times \mathbb{R} \times U$, and in addition $f_{a}^{*}(t, x, u)$ and $L^{*}(t, x, u)$ are Hölder continuous in $t$ with exponent $\beta$, i.e.,

$$
\sup _{u \in U, x \in \mathbb{R}} \sup _{0 \leq s<t \leq T} \frac{|\psi(t, x, u)-\psi(s, x, u)|}{|t-s|^{\beta}} \leq c,
$$


where $\psi=f_{a}^{*}, L^{*}$, and $c$ is a finite constant.

(ii) Under (H3), for $\psi=f_{a}^{*}, L^{*}$, the partial derivatives $\psi_{x}$ and $\psi_{x x}$ are continuous and bounded on $[0, T] \times \mathbb{R} \times U$.

(iii) Under (H4), there exists $c>0$ such that $\sup _{(t, x) \in[0, T] \times \mathbb{R}} \mid f_{a}^{*}(t, x, u)-f_{a}^{*}(t, x$, $\left.u^{\prime}\right)|\leq c| u-u^{\prime} \mid$, for each $a \in \mathcal{A}$, i.e., each $f_{a}^{*}$ is Lipschitz continuous in $u \in U$.

(iv) Under (H5), for any $q \in \mathbb{R}$, the set of minimizers $\arg \min _{u \in U}\left[f_{a}^{*}(t, x, u) q+\right.$ $\left.L^{*}(t, x, u)\right]$ is a singleton.

Proof. (i) We analyze $f_{a}^{*}$ only, and the case for $L^{*}$ is similar. We first prove (15) for $f_{a}^{*}$. Since $\left(\mu_{t}^{1}, \cdots, \mu_{t}^{K}\right) \in \mathcal{M}_{[0, T]}$ with Hölder exponent $\beta$, and since $\mid f_{a}(x, u, y)-$ $f_{a}\left(x, u, y^{\prime}\right)\left|\leq B_{2}\right| y-y^{\prime} \mid$ with the Lipschitz constant $B_{2}$ independent of $(x, u)$, we may select a finite constant $c>0$ such that (15) holds.

The boundedness of $f_{a}^{*}$ is obvious. We take $(t, x, u)$ and $\left(t^{\prime}, x^{\prime}, u^{\prime}\right)$, both from the set $[0, T] \times \mathbb{R} \times U$. We have

$$
\begin{aligned}
& \left|f_{a}^{*}(t, x, u)-f_{a}^{*}\left(t^{\prime}, x^{\prime}, u^{\prime}\right)\right| \\
= & \left|f_{a}^{*}(t, x, u)-f_{a}^{*}\left(t, x^{\prime}, u^{\prime}\right)\right|+\left|f_{a}^{*}\left(t, x^{\prime}, u^{\prime}\right)-f_{a}^{*}\left(t^{\prime}, x^{\prime}, u^{\prime}\right)\right| .
\end{aligned}
$$

By (H2), it is easy to show that $\left|f_{a}(x, u, y)-f_{a}\left(x^{\prime}, u^{\prime}, y\right)\right| \rightarrow 0$ uniformly, as $\left|x-x^{\prime}\right|+$ $\left|u-u^{\prime}\right| \rightarrow 0$. By the definition of $f_{a}^{*}$, this implies that $\left|f_{a}^{*}(t, x, u)-f_{a}^{*}\left(t, x^{\prime}, u^{\prime}\right)\right| \rightarrow 0$ uniformly as $\left|x-x^{\prime}\right|+\left|u-u^{\prime}\right| \rightarrow 0$. By combining (16) with (15), it follows that $\left|f_{a}^{*}(t, x, u)-f_{a}^{*}\left(t^{\prime}, x^{\prime}, u^{\prime}\right)\right| \rightarrow 0$ uniformly, as $\left|t-t^{\prime}\right|+\left|x-x^{\prime}\right|+\left|u-u^{\prime}\right| \rightarrow 0$.

(ii) Under (H3), the partial derivatives $\psi_{x}, \psi_{x x}$ exist, and for $i=1,2$,

$$
\begin{aligned}
& \frac{\partial\left[f_{a}^{*}(t, x, u)\right]^{i}}{\partial x^{i}}=\sum_{k=1}^{K} \pi_{k} \int \frac{\partial\left[f_{a}(x, u, y)\right]^{i}}{\partial x^{i}} \mu_{t}^{k}(d y), \\
& \frac{\partial\left[L^{*}(t, x, u)\right]^{i}}{\partial x^{i}}=\sum_{k=1}^{K} \pi_{k} \int \frac{\partial[L(x, u, y)]^{i}}{\partial x^{i}} \mu_{t}^{k}(d y),
\end{aligned}
$$

where the integration and differentiation are interchangeable due to the boundedness of the derivatives of $f_{a}$ and $L$ in $x$. The continuity of the derivatives may be proved by following similar steps as in (i).

The proof of (iii) is obvious. Since both $f_{a}^{*}$ and $L^{*}$ are defined using the measure $\sum_{i=1}^{K} \pi_{i} \mu_{t}^{i}(d y)$, (iv) follows.

5. HJB Equation for Optimal Control of a Single Agent. The NCE methodology translates into the analysis of the three coupled equations specifying (i) the HJB equation for the optimal control problem based upon a single agent, (ii) the optimal strategy for each type of agents as classified by the dynamic parameter $a$, and (iii) the closed-loop $\mathrm{M}-\mathrm{V}$ equation. Instead of directly analyzing the coupled equation system, we shall begin by dealing with the decoupled individual equations. This will provide insight into the structure of the underlying game problem. 
By using the notation in (13), we write the HJB equation

$$
-\frac{\partial V_{a}}{\partial t}=\inf _{u \in U}\left\{f_{a}^{*}(t, x, u) \frac{\partial V_{a}}{\partial x}+L^{*}(t, x, u)\right\}+\frac{\sigma^{2}}{2} \frac{\partial^{2} V_{a}}{\partial x^{2}}
$$

where the terminal condition is $V_{a}(T, x)=0$. As in Section 4, the measure flow $\left(\mu_{t}^{1}, \cdots, \mu_{t}^{K}\right), 0 \leq t \leq T$, which is involved in $f_{a}^{*}$ and $L^{*}$, is not restricted to satisfy (9)-(11) simultaneously. The first step is to identify suitable conditions, so that the HJB equation (17) gives a unique classical solution where the measure flow is only generally specified.

Let $Q_{T}=(0, T) \times \mathbb{R}$ and $\bar{Q}_{T}=[0, T] \times \mathbb{R}$. We denote by $C^{1,2}\left(\bar{Q}_{T}\right)$ (resp., $\left.C^{1,2}\left(Q_{T}\right)\right)$ the set of continuous functions $v(t, x)$ with continuous derivatives $v_{t}, v_{x x}$ on $\bar{Q}_{T}$ (resp., $Q_{T}$ ). Let $C_{b}^{1,2}\left(\bar{Q}_{T}\right)$ be the set consisting of all bounded functions in $C^{1,2}\left(\bar{Q}_{T}\right)$.

ThEOREM 5. Suppose (H1)-(H4) hold, and the measure flow $\left(\mu_{t}^{1}, \cdots, \mu_{t}^{K}\right), 0 \leq$ $t \leq T$ is in the class $\mathcal{M}_{[0, T]}$ with Hölder exponent $\beta \in(0,1]$. Then equation (17) has a unique solution $V_{a} \in C_{b}^{1,2}\left(\bar{Q}_{T}\right)$.

Proof. We give the proof by standard methods in optimal control of non-degenerate diffusion processes. First, we can follow exactly the argument for proving Theorem 6.2 in Appendix of [15] to show that there exists a solution (actually determined as a continuous function on $\left.\bar{Q}_{T}\right) V_{a} \in C^{1,2}\left(Q_{T}\right)$ by only assuming that both $f_{a}^{*}$ and $L^{*}$ satisfy Hölder continuity in $t$ rather than have a continuous derivative in $t$ [15]. In constructing this particular solution $V_{a}$ by the approximation procedure, we may obtain an a priori constant upper bound for $V_{a}$ by use of the boundedness of $f_{a}^{*}$ and $L^{*}$.

For any $R>0$, let $B_{R}=(-R, R)$ and $\bar{B}_{R}=[-R, R]$. Subsequently, we can show by standard Hölder estimate [15] (pp. 207-208) that for a small positive constant $\delta \in(0,1), V_{a} \in C^{1+\delta / 2,2+\delta}\left((0, T) \times B_{R}\right)$ by restricting $V_{a}$ to the domain $(0, T) \times B_{R}$. In other words, under the parabolic distance $d((t, x),(s, y))=\left(|t-s|+|x-y|^{2}\right)^{1 / 2}$, the functions $V_{a},\left(V_{a}\right)_{t}$ and $\left(V_{a}\right)_{x x}$ are all Hölder continuous on $(0, T) \times B_{R}$ with exponent $\delta$. This further implies the first and second order derivatives of $V_{a}$ appearing in the HJB equation may be extended to $[0, T] \times \bar{B}_{R}$ in an obvious way and $V_{a} \in$ $C^{1+\delta / 2,2+\delta}\left([0, T] \times \bar{B}_{R}\right)$. Since $R$ is arbitrary, we have $V_{a} \in C^{1,2}\left(\bar{Q}_{T}\right)$.

The uniqueness follows from the standard verification theorem by interpreting $V_{a}$ as the value function of an associated stochastic optimal control problem. This completes the proof.

As in [15], by the verification theorem we may obtain uniqueness in the wider class $C_{p}^{1,2}\left(Q_{T}\right) \cap C\left(\bar{Q}_{T}\right)$ (i.e., when $v \in C\left(\bar{Q}_{T}\right)$ is restricted to $Q_{T}$, it is also in $C_{p}^{1,2}\left(Q_{T}\right)$ ) where $C_{p}^{1,2}\left(Q_{T}\right) \subset C^{1,2}\left(Q_{T}\right)$ consists of functions satisfying a polynomial growth in the spatial variable $x$. 
Given a measure flow in $\mathcal{M}_{[0, T]}$, by Theorem 5 we obtain a smooth solution for $V_{a}$ and subsequently, corresponding to each $(t, x) \in[0, T] \times \mathbb{R}$, under $(\mathrm{H} 5)$ we get $u(t, x)$ as a well defined function minimizing the right hand side of (9). Hence we write the optimal control law in a feedback form

$$
u=\varphi_{a}\left(t, x \mid \mu_{.}^{1}, \cdots, \mu_{.}^{K}\right)
$$

where $(t, x) \in[0, T] \times \mathbb{R},\left(\mu_{t}^{1}, \cdots, \mu_{t}^{K}\right) \in \mathcal{M}_{[0, T]}$, and $a$ is the dynamic parameter for the associated agent.

We introduce an additional assumption for the control law (18).

(H6) For each $a \in \mathcal{A}$ and $\left(\mu_{t}^{1}, \cdots, \mu_{t}^{K}\right) \in \mathcal{M}_{[0, T]}$, the function $\varphi_{a}\left(t, x \mid \mu_{.}^{1}, \cdots, \mu^{K}\right)$ is continuous in $(t, x) \in[0, T] \times \mathbb{R}$, and Lipschitz continuous in $x \in \mathbb{R}$.

Note that to explicitly verify the Lipschitz continuity in $x$ for the feedback control law, we usually need more concrete assumptions, such as affine linearity in $u_{i}$ for the dynamics of the $i$ th agent, combined with smoothness and convexity of the cost integrand with respect to the control. In the literature, Lipschitz continuity of the feedback has been a well studied topic; see, e.g., [15].

Denote $\mu_{t}^{o}=\left(\mu_{t}^{1}, \cdots, \mu_{t}^{K}\right) \in \mathcal{M}_{[0, T]}$ where $0 \leq t \leq T$. By use of (18) we may write a vector of feedback control laws with $K$ distinct values of $a$. As in (12), for $a=\theta_{i}$ we will simply write $\varphi_{a}$ as $\varphi_{i}$, and consequently we define the following map from $\mathcal{M}_{[0, T]}$ to the $K$-fold product set $\left[C\left(\bar{Q}_{T}\right)\right]^{K}$

$$
\Gamma\left(\mu_{.}^{o}\right)=\left(\varphi_{1}\left(t, x \mid \mu_{.}^{o}\right), \cdots, \varphi_{K}\left(t, x \mid \mu_{.}^{o}\right)\right)
$$

where $\mu_{\text {. }}^{o}=\left(\mu_{.}^{1}, \cdots, \mu^{K}\right)$ and we use the same argument $x$ inside each component function. When these individual control laws are used by the corresponding agents, the variable $x$ is substituted by its own state variable (see (12)).

The nonlinear map $\Gamma$ gives an important characterization of the individual-mass interaction, and it turns out to have a close relation to the well known best response map in noncooperative game theory. In a static $n$-person noncooperative game [17], once the actions for the other $n-1$ players are assumed, the best response map of the given player will determine its optimal choice of one or more actions which is optimal conditioned on other agents' actions assumed in the first place. In our large population multi-class agent game model, we may view the $K$-tuple $\mu_{.}^{o}=\left(\mu_{.}^{1}, \cdots, \mu^{K}\right)$ as the effect of a virtual player, and then each $\varphi_{i}$ may be regarded as the local optimal strategy in response to that given $\mu_{\text {. }}^{o}$. For this reason, we just extend the conventional name by calling $\Gamma$ the best response map.

6. The M-V Equation with Decentralized Lipschitz Feedback - We recall that in (11) the feedback control has a functional dependence on the measure flow $\left(\mu_{t}^{1}, \cdots, \mu_{t}^{K}\right), 0 \leq t \leq T$. That causes the M-V equation (11) to be coupled with the 
HJB equation (9). In this section and in parallel to the treatment in Section 5, we proceed by assuming a known feedback control law $\phi_{a}(t, x)$ (which may be written as $\phi_{k}(t, x)$ when $\left.a=\theta_{k}\right)$ for the associated agents with dynamic parameter $a$, which leads to the following auxiliary equation

$$
\left\{\begin{aligned}
d x_{1} & =f_{1}\left[x_{1}, \phi_{1}\left(t, x_{1}\right), \mu_{t}^{1}, \cdots, \mu_{t}^{K}\right] d t+\sigma d w_{1}, \\
& \vdots \\
d x_{K} & =f_{K}\left[x_{K}, \phi_{K}\left(t, x_{K}\right), \mu_{t}^{1}, \cdots, \mu_{t}^{K}\right] d t+\sigma d w_{K},
\end{aligned}\right.
$$

where the function $\phi_{k}(t, x)$ is the class $C_{L i p(x)}\left(\bar{Q}_{T}, U\right)$ consisting of continuous functions from $\bar{Q}_{T}=[0, T] \times \mathbb{R}$ to $U$, which are Lipschitz continuous in $x$. The initial conditions and the Brownian motions in (20) are specified in the same form as in (12). The key question is whether there exists a well defined vector process $\left(x_{1}, \cdots, x_{K}\right)$ satisfying equation (20) in the McKean-Vlasov sense.

Before establishing existence results for (20), we introduce some preliminary material about the metric on a space of probability measures. For related treatment, the reader is referred to [44] (pp.172-174). Let $C\left([0, T], \mathbb{R}^{K}\right)$ be the space of continuous functions on $[0, T]$ and we write it in the abbreviated form $C^{(K)}$. For $x, y \in C\left([0, T], \mathbb{R}^{K}\right)$, define the norm $\|x-y\|=\sup _{t \in[0, T]}|x(t)-y(t)|$. Then $\left(C^{(K)},\|\cdot\|\right)$ constitutes a Banach space. Also, we introduce the metric $\rho(x, y)=$ $\sup _{t \in[0, T]}|x(t)-y(t)| \wedge 1$, and it is easy to verify that $\left(C^{(K)}, \rho\right)$ forms a complete metric space, i.e., each Cauchy sequence has a limit in $\left(C^{(K)}, \rho\right)$. It is well known in functional analysis that $\left(C^{(K)},\|\cdot\|\right)$ is a separable space. On the other hand, the convergence of a sequence in $\left(C^{(K)},\|\cdot\|\right)$ is equivalent to its convergence in the metric $\rho$. Hence $\left(C^{(K)}, \rho\right)$ is a separable and complete metric space.

On $C\left([0, T], \mathbb{R}^{K}\right)$, we specify the $\sigma$-algebra $\mathcal{F}^{(K)}$ induced by all cylindrical sets of the form $\left\{x(\cdot) \in C^{(K)}: x_{t_{i}} \in B_{i}, t_{i} \in[0, T], i=1,2, \cdots, l\right\}$ where each $B_{i}$ is a Borel set in $\mathbb{R}^{K}$ and $l$ is any positive integer. Let $\mathcal{M}\left(C^{(K)}\right)$ be the space of all probability measures $m$ on $\left(C^{(K)}, \mathcal{F}^{(K)}\right)$. Thus each $\left(C^{(K)}, \mathcal{F}^{(K)}, m\right)$ is a probability space. For the product space $C^{(K)} \times C^{(K)}$, the space of probability measures is defined in an obvious manner, and denoted as $\mathcal{M}\left(C^{(K)} \times C^{(K)}\right)$. We introduce the canonical process $X$ to be a random process with the sample space $C^{(K)}$, i.e., $X_{t}(\omega)=\omega_{t}$ for $\omega \in C^{(K)}$.

By the same procedure but replacing the dimension number $K$ by one, we may define the space of probability measures on $C([0, T], \mathbb{R})$, and we denote it by $\mathcal{M}\left(C^{(1)}\right)$.

Based upon the metric $\rho$ in $\left(C^{(K)}, \rho\right)$, we now introduce the so-called Vasershtein metric on $\mathcal{M}\left(C^{(K)}\right)$. For $m_{1}, m_{2} \in \mathcal{M}\left(C^{(K)}\right)$, define

$$
D_{T}\left(m_{1}, m_{2}\right)=\inf _{m} \int_{C^{(K)} \times C^{(k)}}\left(\sup _{s \leq T}\left|X_{s}\left(\omega_{1}\right)-X_{s}\left(\omega_{2}\right)\right| \wedge 1\right) d m\left(\omega_{1}, \omega_{2}\right),
$$

where $m \in \mathcal{M}\left(C^{(K)} \times C^{(K)}\right), p_{1} \circ m=m_{1}$ and $p_{2} \circ m=m_{2}$ denoting the two marginal 
distributions of $m$, and $m$ is called a coupling of $m_{1}$ and $m_{2}$. This gives a complete metric on $\mathcal{M}\left(C^{(K)}\right)$, which implies each fundamental sequence $\left\{m_{i}, i \geq 1\right\}$ under the metric $D_{T}$ has a limit in $\mathcal{M}\left(C^{(K)}\right)$ [44]. We note that for both $C^{(K)}$ and $\mathcal{M}\left(C^{(K)}\right)$, we may define the corresponding space on a smaller interval $[0, t] \subset[0, T]$. Similarly, $D_{t}\left(m_{1}, m_{2}\right)$ may be defined for $t<T$ by considering the restriction of $m_{i} \in \mathcal{M}\left(C^{(K)}\right)$ to $C\left([0, t], \mathbb{R}^{K}\right)$, which is embedded in a natural way as a subspace of $C\left([0, T], \mathbb{R}^{K}\right)$.

Remark. If $(\cdot \wedge 1)$ in $(21)$ is replaced by $(\cdot \wedge d)$ for $d>0$, we may denote the associated metric by $D_{T}^{d}\left(m_{1}, m_{2}\right)$, and all analysis in this section still holds.

Let $m \in \mathcal{M}\left(C^{(K)}\right)$ and define the random process $y=\left(y_{1}, \cdots, y_{K}\right)$ on $[0, T]$ as follows.

$$
y_{i}(t)=x_{i}(0)+\sigma w_{i}(t)+\int_{0}^{t} \int_{C^{(K)}} \sum_{j=1}^{K} \pi_{j} f_{i}\left(y_{i}(s), \phi_{i}\left(s, y_{i}(s)\right), \omega_{s}^{j}\right) d m(\omega) d s
$$

where $0 \leq t \leq T$ and the canonical process is denoted as $\omega .=\left(\omega^{1}, \cdots, \omega^{K}\right)$. We denote the law of $y$ on $[0, T]$ by $\Phi(m)$ which clearly belongs to $\mathcal{M}\left(C^{(K)}\right)$. The existence proof below is based upon a fixed point method to show that there exists a unique $m$ such that $\Phi(m)=m$, and we finish the proof by determining $m$ as a product form.

THEOREM 6. Under (H1)-(H6), there is a unique consistent solution pair $\left(x_{1}, \cdots\right.$, $\left.x_{K}\right)$ and $\left(\mu^{1}, \cdots, \mu^{K}\right)$ to (20).

Proof. We break the proof into two steps where the first step is similar to the proof of Theorem 1.1 in [44] which deals with a conventional M-V equation for a single class of particles. For the reader's convenience, we give the details of the fixed point analysis.

Step 1. Take $m, \hat{m} \in \mathcal{M}\left(C^{(K)}\right)$, and let $y$ be defined by (22). Similarly, $\hat{y}$ is defined by (22) after replacing $m$ by $\hat{m}$. Both $y_{i}$ and $\hat{y}_{i}$ have the same initial condition $x_{i}(0)$, $1 \leq i \leq K$. Denote

$$
\begin{aligned}
\zeta\left(s, y_{i}(s), \omega_{s}\right) & =\sum_{j=1}^{K} \pi_{j} f_{i}\left(y_{i}(s), \phi_{i}\left(s, y_{i}(s)\right), \omega_{s}^{j}\right), \\
\hat{\zeta}\left(s, \hat{y}_{i}(s), \omega_{s}\right) & =\sum_{j=1}^{K} \pi_{j} f_{i}\left(\hat{y}_{i}(s), \phi_{i}\left(s, \hat{y}_{i}(s)\right), \omega_{s}^{j}\right),
\end{aligned}
$$

where $0 \leq s \leq T$. It follows that

$$
\sup _{0 \leq s \leq t}\left|y_{i}(s)-\hat{y}_{i}(s)\right| \leq \int_{0}^{t}\left|\int_{C^{(K)}} \zeta\left(s, y_{i}(s), \omega_{s}\right) d m(\omega)-\int_{C^{(K)}} \hat{\zeta}\left(s, \hat{y}_{i}(s), \omega_{s}\right) d \hat{m}(\omega)\right| d s .
$$


For any $\bar{m} \in \mathcal{M}\left(C^{(K)} \times C^{(K)}\right)$ such that $p_{1} \circ \bar{m}=m$ and $p_{2} \circ \bar{m}=\hat{m}$, we have

$$
\begin{aligned}
\xi_{s} & \triangleq\left|\int_{C^{(K)}} \zeta\left(s, y_{i}(s), \omega_{s}\right) d m(\omega)-\int_{C^{(K)}} \hat{\zeta}\left(s, \hat{y}_{i}(s), \omega_{s}\right) d \hat{m}(\omega)\right| \\
& =\left|\int_{C^{(K)} \times C^{(K)}} \zeta\left(s, y_{i}(s), \omega_{s}\right) d \bar{m}(\omega, \hat{\omega})-\int_{C^{(K)} \times C^{(K)}} \hat{\zeta}\left(s, \hat{y}_{i}(s), \hat{\omega}_{s}\right) d \bar{m}(\omega, \hat{\omega})\right| \\
& \leq C_{1}\left(\left|y_{i}(s)-\hat{y}_{i}(s)\right| \wedge 1\right)+\sum_{j} \int_{C^{(K)} \times C^{(K)}} C_{2}\left(\left|\omega_{s}^{j}-\hat{\omega}_{s}^{j}\right| \wedge 1\right) d \bar{m}(\omega, \hat{\omega}),
\end{aligned}
$$

where $C_{1}$ and $C_{2}$ are two constants obtained from the boundedness and Lipschitz continuity of both $f_{i}$ and $\phi_{i}$. Clearly, for all trajectories of the canonical process, we have

$$
\sum_{j}\left(\left|\omega_{s}^{j}-\hat{\omega}_{s}^{j}\right| \wedge 1\right) \leq K\left(\left|\omega_{s}-\hat{\omega}_{s}\right| \wedge 1\right)
$$

Hence it follows from (24) and (25) that

$$
\xi_{s} \leq C_{1}\left(\left|y_{i}(s)-\hat{y}_{i}(s)\right| \wedge 1\right)+\int_{C^{(K)} \times C^{(K)}} C_{2}\left(\left|\omega_{s}-\hat{\omega}_{s}\right| \wedge 1\right) d \bar{m}(\omega, \hat{\omega}),
$$

which implies

$$
\xi_{s} \leq C_{1}\left(\left|y_{i}(s)-\hat{y}_{i}(s)\right| \wedge 1\right)+C_{2} D_{s}(m, \hat{m})
$$

since $\bar{m}$ is any coupling of $m$ and $\hat{m}$. Consequently, it follows from (23) and (26) that

$$
\sup _{0 \leq s \leq t}\left|y_{i}(s)-\hat{y}_{i}(s)\right| \leq \int_{0}^{t}\left[C_{1}\left(\left|y_{i}(s)-\hat{y}_{i}(s)\right| \wedge 1\right)+C_{2} D_{s}(m, \hat{m})\right] d s .
$$

Then (27) combined with Gronwall's lemma gives

$$
\sup _{0 \leq s \leq t}\left|y_{i}(s)-\hat{y}_{i}(s)\right| \wedge 1 \leq C_{T} \int_{0}^{t} D_{s}(m, \hat{m}) d s
$$

where the constant $C_{T}$ depends upon the terminal time $T$. Subsequently,

$$
\sup _{0 \leq s \leq t}|y(s)-\hat{y}(s)| \wedge 1 \leq K C_{T} \int_{0}^{t} D_{s}(m, \hat{m}) d s .
$$

We see that $y$ and $\hat{y}$ induce two probability distributions, denoted as $\Phi(m)$ and $\Phi(\hat{m})$, respectively, on $C^{(K)}$, and furthermore, the joint distribution of $(y, \hat{y})$ gives a measure $\bar{m}_{\Phi}$ on $C^{(K)} \times C^{(K)}$. By taking expectation on the left hand side of (29) and expressing the calculation in terms of the canonical process and $\bar{m}_{\Phi} \in \mathcal{M}\left(C^{(K)} \times\right.$ $\left.C^{(K)}\right)$, we get an upper bound for $D_{t}(\Phi(m), \Phi(\hat{m}))$. This leads to the inequality

$$
D_{t}\left(\Phi\left(m_{1}\right), \Phi\left(m_{2}\right)\right) \leq K C_{T} \int_{0}^{t} D_{s}\left(m_{1}, m_{2}\right) d s .
$$


Now, following a similar argument as in [44], we can show that $\left\{\Phi^{k}(m), k \geq 1\right\}$ forms a fundamental sequence, and there exists a unique $m \in \mathcal{M}\left(C^{(K)}\right)$ satisfying $\Phi(m)=m$. This completes the first step.

Step 2. Next we examine the structure of the fixed point probability measure $m$ arising in the existence proof for a solution of (20). Let $x=\left(x_{1}, \cdots, x_{K}\right)$ denote the solution of $(22)$ on $[0, T]$ when $m=\Phi(m)$. Since all $x_{i}$ have independent initial conditions and Brownian motions, they form $n$ independent processes. We denote product $B_{i}=B_{i}^{1} \times \cdots \times B_{i}^{K}$, where $i=1,2, \cdots$, and each $B_{i}^{k}$ is a Borel set in $\mathbb{R}$. Since $m$ is the law of the associated solution $x$ on $[0, T]$ which has $K$ independent components, we have

$$
\begin{aligned}
m\left(\omega_{t_{i}} \in B_{i}, i=1,2, \cdots, l\right) & =P\left(x_{1}\left(t_{i}\right) \in B_{i}^{1}, \cdots, x_{K}\left(t_{i}\right) \in B_{i}^{K}, i=1,2 \cdots, l\right) \\
& =\prod_{k=1}^{K} P\left(x_{k}\left(t_{i}\right) \in B_{i}^{k}, i=1,2, \cdots, l\right) \\
& =\prod_{k=1}^{K} m^{i}\left(\omega_{t_{i}}^{k} \in B_{i}^{k}, i=1,2, \cdots, l\right)
\end{aligned}
$$

where $m^{k}$ is the law of $x_{k}, 1 \leq k \leq K$. We get the second equality by independence in terms of the underlying probability space $(\Omega, \mathcal{F}, P)$. Since $(31)$ holds for all product Borel sets in $\mathbb{R}^{K}$, we see that $m=m^{1} \times \cdots \times m^{K}$ on $\mathcal{F}^{(K)}$.

Now for $m=m^{1} \times \cdots \times m^{K}$, equation (22) for $x_{i}$ reduces to

$$
\begin{aligned}
x_{i}(t) & =x_{i}(0)+\sigma w_{i}(t)+\int_{0}^{t} \int_{C^{(K)}} \sum_{j} \pi_{j} f_{i}\left(x_{i}(s), \phi_{i}\left(s, x_{i}(s)\right), \omega_{s}^{j}\right) d m(\omega) d s \\
& =x_{i}(0)+\sigma w_{i}(t)+\int_{0}^{t} \sum_{j} \pi_{j} \int_{C([0, T], \mathbb{R})} f_{i}\left(x_{i}(s), \phi_{i}\left(s, x_{i}(s)\right), \omega_{s}^{j}\right) d m^{j}\left(\omega^{j}\right) d s \\
& =x_{i}(0)+\sigma w_{i}(t)+\int_{0}^{t} \int_{\mathbb{R}} \sum_{j} \pi_{j} f_{i}\left(x_{i}(s), \phi_{i}\left(s, x_{i}(s)\right), y\right) \mu_{s}^{j}(d y) d s
\end{aligned}
$$

where $\mu_{s}^{j}$ is the marginal distribution of $m^{j}$ at time $s$. We may further express the right hand side of $(22)$ in terms of the product measure $\mu_{t}^{1} \times \cdots \times \mu_{t}^{K}$ which is the marginal distribution at time $t$ for the law of $x$, reducing (22) to the form (20); uniqueness of the consistent pair follows from Step 1. This completes the proof.

For a set of functions $\left(\phi_{1}, \cdots, \phi_{K}\right)$ such that $\phi_{k} \in C_{L i p(x)}\left(\bar{Q}_{T}, U\right)$, for $1 \leq k \leq K$, we implement it as the set of control laws in (20), which leads to a well defined solution $x=\left(x_{1}, \cdots, x_{K}\right)$ on $[0, T]$ by Theorem 6 . Let the law of the resulting solution $x$ be denoted by $m$, and define the map from $\left(C_{\operatorname{Lip}(x)}\left(\bar{Q}_{T}, U\right)\right)^{K}$ to $\mathcal{M}\left(C^{(K)}\right)$ :

$$
m=\widehat{\Gamma}\left(\phi_{1}, \cdots, \phi_{K}\right)
$$


By taking marginal distributions for the solution process or equivalently for $m$ in (32), we get a measure flow $\left(\mu_{t}^{1}, \cdots, \mu_{t}^{K}\right), 0 \leq t \leq T$. We introduce the map:

$$
\left(\mu_{.}^{1}, \cdots, \mu_{.}^{K}\right)=\bar{\Gamma}\left(\phi_{1}, \cdots, \phi_{K}\right) .
$$

Each $\mu_{.}^{k}$ on the left hand side corresponds to all marginal distributions on $[0, T]$ of the component $x_{t}^{k}, 0 \leq t \leq T$. It is easy to see that there is a well defined map relating $\left(\mu_{.}^{1}, \cdots, \mu_{.}^{K}\right)$ to $m$ :

$$
\left(\mu_{.}^{1}, \cdots, \mu_{.}^{K}\right)=p_{\mu}(m),
$$

where $m \in \widehat{\Gamma}\left(\left[C_{L i p(x)}\left(\bar{Q}_{T}, U\right)\right]^{K}\right)$. We will call $p_{\mu}$ the projection map.

Lemma 7. Under the assumptions in Theorem 6, for any $\left(\phi_{1}, \cdots, \phi_{K}\right)$ such that $\phi_{k} \in C_{L i p(x)}\left(\bar{Q}_{T}, U\right)$, for $1 \leq k \leq K$, the measure flow $\left(\mu_{t}^{1}, \cdots, \mu_{t}^{K}\right), 0 \leq t \leq T$, obtained from (33) is in the class $\mathcal{M}_{[0, T]}$. Hence $\bar{\Gamma}$ is a map from $\left(C_{\operatorname{Lip}(x)}\left(\bar{Q}_{T}, U\right)\right)^{K}$ to $\mathcal{M}_{[0, T]}$.

Proof. By Theorem 6, there exists a unique solution $x_{t}=\left(x_{t}^{1}, \cdots, x_{t}^{K}\right), 0 \leq t \leq T$, to $(20)$ when the set of control laws $\left(\phi_{1}, \cdots, \phi_{K}\right)$ is used. Take any $0 \leq t^{\prime}<t^{\prime \prime} \leq T$. For any bounded and Lipschitz continuous function $\psi(y)$ with a Lipschitz constant $\operatorname{Lip}(\psi)$, we have

$$
\begin{aligned}
\left|\int \psi(y) \mu_{t^{\prime}}^{j}(d y)-\int \psi(y) \mu_{t^{\prime \prime}}^{j}(d y)\right| & =\left|E \psi\left(x_{t^{\prime}}^{j}\right)-E \psi\left(x_{t^{\prime \prime}}^{j}\right)\right| \\
& \leq \operatorname{Lip}(\psi) E\left|x_{t^{\prime}}^{j}-x_{t^{\prime \prime}}^{j}\right| .
\end{aligned}
$$

On the other hand, we have

$$
x_{t^{\prime \prime}}^{j}=x_{t^{\prime}}^{j}+\int_{t^{\prime}}^{t^{\prime \prime}} f_{j}\left[x_{s}^{j}, \phi_{j}\left(s, x_{s}^{j}\right), \mu_{t}^{1}, \cdots, \mu_{t}^{K}\right] d s+\sigma\left(w_{j}\left(t^{\prime \prime}\right)-w_{j}\left(t^{\prime}\right)\right),
$$

and it follows that

$$
E\left|x^{j}\left(t^{\prime \prime}\right)-x^{j}\left(t^{\prime}\right)\right|^{2} \leq 2 C_{1}^{2}\left|t^{\prime \prime}-t^{\prime}\right|^{2}+2 \sigma^{2}\left|t^{\prime \prime}-t^{\prime}\right|,
$$

where $C_{1}$ is an upper bound for $f_{j}(x, u, y)$. Hence

$$
\begin{aligned}
\left|\int \psi(y) \mu_{t^{\prime}}^{j}(d y)-\int \psi(y) \mu_{t^{\prime \prime}}^{j}(d y)\right| & \leq \sqrt{2} \operatorname{Lip}(\psi)\left(C_{1}\left|t^{\prime}-t^{\prime \prime}\right|+|\sigma|\left|t^{\prime}-t^{\prime \prime}\right|^{1 / 2}\right) \\
& \leq \sqrt{2} \operatorname{Lip}(\psi)\left(C_{1} \sqrt{T}+|\sigma|\right)\left|t^{\prime}-t^{\prime \prime}\right|^{1 / 2}
\end{aligned}
$$

for all $t^{\prime}, t^{\prime \prime} \in[0, T]$. By the arbitrariness of $\psi$ and $j$, the lemma follows.

The implication of this lemma is that, after all agents apply Lipschitz control laws determined by their own parameter type, the resulting measure flow maintains a certain continuity. Consequently, one can obtain a well defined new strategy by solving an HJB equation involving that measure flow. Such a procedure makes it feasible to develop strategy or policy iteration by use of the population limit. In other words, one may repeatedly apply the two operators $\Gamma$ and $\bar{\Gamma}$ alternatively. 
7. Feedback Regularity and Strategy Revision. We have the following proposition about the composite map formed by $\bar{\Gamma}$ and $\Gamma$.

Proposition 8. Assume (H1)-(H6) and let $\mu_{t}^{o}=\left(\mu_{t}^{1}, \cdots, \mu_{t}^{K}\right) \in M_{[0, T]}$. We have $\bar{\Gamma} \circ \Gamma\left(\mu_{.}^{o}\right) \in M_{[0, T]}$, i.e., $\Gamma_{\mathcal{M}} \triangleq \bar{\Gamma} \circ \Gamma$ is a map from $\mathcal{M}_{[0, T]}$ to $\mathcal{M}_{[0, T]}$.

Proof. Given $\mu_{t}^{o} \in \mathcal{M}_{[0, T]}$, we obtain a set of well defined feedback control laws $\left(\phi_{1}, \cdots, \phi_{K}\right)$ by the results in Section 5. Subsequently, the Lipschitz feedback assumption combined with Lemma 7 implies this proposition.

Now it is clear that we obtain a solution to the equation system (9)-(11) for the NCE methodology if we can find $\mu_{t}^{o} \in \mathcal{M}_{[0, T]}$ to satisfy the fixed point equation

$$
\bar{\Gamma} \circ \Gamma\left(\mu_{.}^{o}\right)=\mu_{.}^{o} .
$$

Here we need to restrict the solution to the set $\mathcal{M}_{[0, T]}$ so that the machinery of the decoupled HJB equation and McKean-Vlasov equation approach may be employed. This is not an essential restriction since once there indeed exists a solution $\mu_{t}^{o}$, we can derive from (11) that $\mu_{t}^{o} \in \mathcal{M}_{[0, T]}$ only by use of the boundedness of $f_{i}(x, u, y)$, $1 \leq i \leq K$.

However, a drawback of directly analyzing (35) is that we only know $\mu_{t}^{o}$ is a "flow" of measures with limited structural information. This makes it difficult to develop fixed point analysis. For this reason, we will use an embedding strategy by finding a certain measure $m \in \mathcal{M}\left(C^{(K)}\right)$, which is associated with $\mu^{o}$. via the projection map (34).

Under (H1)-(H6), for any $\mu_{.}^{o} \in \mathcal{M}_{[0, T]}$, we obtain a set of control laws $\Gamma\left(\mu_{.}^{o}\right)=$ $\left(\phi_{\theta_{1}}, \cdots, \phi_{\theta_{K}}\right)$ by solving the HJB equation (17). Subsequently we get $m=\widehat{\Gamma} \circ \Gamma\left(\mu^{o}\right) \in$ $\mathcal{M}\left(C^{(K)}\right)$, which further induces $p_{\mu} \circ \widehat{\Gamma} \circ \Gamma\left(\mu_{.}^{o}\right) \in \mathcal{M}_{[0, T]}$. Now we introduce an auxiliary fixed point equation

$$
\widehat{\Gamma} \circ \Gamma \circ p_{\mu}(m)=m,
$$

where $m$ belongs to the image $\widehat{\Gamma} \circ \Gamma\left(\mathcal{M}_{[0, T]}\right) \subset \mathcal{M}\left(C^{(K)}\right)$ of the map $\widehat{\Gamma} \circ \Gamma$ on $\mathcal{M}_{[0, T]}$.

For analyzing equation (36), we introduce the regularity condition as follows. Take two measures $m, \tilde{m} \in \widehat{\Gamma} \circ \Gamma\left(\mathcal{M}_{[0, T]}\right)$. Denote the two measure flows $\mu_{.}^{o}=p_{\mu}(m)$ and $\tilde{\mu}^{o}=p_{\mu}(\tilde{m})$ on $[0, T]$. We write the associated drift vector at time $t$ as $f_{a}\left[x_{t}, u_{t}, \mu_{t}^{o}\right]$ and $f_{a}\left[x_{t}, u_{t}, \tilde{\mu}_{t}^{o}\right]$. The cost integrands are given as $L\left[x_{t}, u_{t}, \mu_{t}^{o}\right]$ and $L\left[x_{t}, u_{t}, \tilde{\mu}_{t}^{o}\right]$. Accordingly, the associated HJB equations (17) are solved to give two sets of feedback control laws $\phi_{a}(t, x)$ and $\tilde{\phi}_{a}(t, x), a \in \mathcal{A}$, with each individual control law being in $C_{L i p(x)}\left(\bar{Q}_{T}, U\right)$ under $(\mathrm{H} 6)$. For instance, we have $\left(\phi_{\theta_{1}}, \cdots, \phi_{\theta_{K}}\right)=\Gamma \circ p_{\mu}(m)$. The feedback regularity (FR) condition is given as: There exists a constant $c_{1}>0$ such that

$$
\sup _{(t, x) \in[0, T] \times \mathbb{R}}\left|\phi_{a}(t, x)-\tilde{\phi}_{a}(t, x)\right| \leq c_{1} D_{T}(m, \tilde{m}), \quad \forall a \in \mathcal{A} .
$$


The FR condition (37) characterizes a sensitivity property for the control law when a perturbation of the pre-image of the measure flow, associated with the map $p_{\mu}$, is involved. This, in turn, is related to certain continuous dependence of the solution $V_{a}$ (as well as its derivatives) to the HJB equation (17) on the functions $f_{a}^{*}$ and $L^{*}$ appearing there.

Lemma 9. Under (H1)-(H6), there exists a constant $c_{2}$ such that

$$
D_{T}(m, \tilde{m}) \leq c_{2} \sup _{a \in \mathcal{A},(t, x) \in \bar{Q}_{T}}\left|\phi_{a}(t, x)-\tilde{\phi}_{a}(t, x)\right|
$$

where $\bar{Q}_{T}=[0, T] \times \mathbb{R}$, and $m, \tilde{m} \in \mathcal{M}\left(C^{(K)}\right)$ are induced by (32) using the two sets of control laws $\phi_{a}$ and $\tilde{\phi}_{a}, a \in \mathcal{A}$, respectively.

Proof. Recall that for $a_{j}=\theta_{k}$, we write $f_{a_{j}}$ (resp., $\phi_{a_{j}}$ ) as $f_{k}$ (resp., $\phi_{k}$ ). For the two solutions, denoted by $x_{t}$ and $\tilde{x}_{t}$, we write their equations by components,

$$
\begin{aligned}
& x_{i}(t)=x_{i}(0)+\sigma w_{i}(t)+\int_{0}^{t} \int_{C^{K}} \sum_{j=1}^{K} \pi_{j} f_{i}\left(x_{i}(s), \phi_{i}\left(s, x_{i}(s)\right), \omega_{s}^{j}\right) d m(\omega) d s, \\
& \tilde{x}_{i}(t)=x_{i}(0)+\sigma w_{i}(t)+\int_{0}^{t} \int_{C^{K}} \sum_{j=1}^{K} \pi_{j} f_{i}\left(\tilde{x}_{i}(s), \tilde{\phi}_{i}\left(s, \tilde{x}_{i}(s)\right), \omega_{s}^{j}\right) d \tilde{m}(\omega) d s,
\end{aligned}
$$

where the initial condition is $x_{i}(0), 1 \leq i \leq K$, and the Brownian motion is $w_{i}$. By use of the Lipschitz continuity of both $f_{i}$ and the feedback control laws, we get

$$
\begin{aligned}
& \left|f_{i}\left(x_{i}(s), \phi_{i}\left(s, x_{i}(s)\right), \omega_{s}^{j}\right)-f_{i}\left(\tilde{x}_{i}(s), \tilde{\phi}_{i}\left(s, \tilde{x}_{i}(s)\right), \tilde{\omega}_{s}^{j}\right)\right| \\
\leq & \left|f_{i}\left(x_{i}(s), \phi_{i}\left(s, x_{i}(s)\right), \omega_{s}^{j}\right)-f_{i}\left(\tilde{x}_{i}(s), \phi_{i}\left(s, \tilde{x}_{i}(s)\right), \tilde{\omega}_{s}^{j}\right)\right| \\
& +\left|f_{i}\left(\tilde{x}_{i}(s), \phi_{i}\left(s, \tilde{x}_{i}(s)\right), \tilde{\omega}_{s}^{j}\right)-f_{i}\left(\tilde{x}_{i}(s), \tilde{\phi}_{i}\left(s, \tilde{x}_{i}(s)\right), \tilde{\omega}_{s}^{j}\right)\right| \\
(39) \leq & C_{1}\left(\left|x_{i}(s)-\tilde{x}_{i}(s)\right| \wedge 1\right)+C_{2} \sup _{(s, x) \in \bar{Q}_{T}}\left|\phi_{i}(s, x)-\tilde{\phi}_{i}(s, x)\right|+C_{3}\left(\left|\omega_{s}^{j}-\tilde{\omega}_{s}^{j}\right| \wedge 1\right) .
\end{aligned}
$$

Now, similar to the derivation of (27), we use (39) to obtain

$$
\begin{aligned}
|x(t)-\tilde{x}(t)| & \leq C_{1} \int_{0}^{t}(|x(s)-\tilde{x}(s)| \wedge 1) d s+C_{2} t \sup _{a \in \mathcal{A},(s, x) \in \bar{Q}_{T}}\left|\phi_{a}-\tilde{\phi}_{a}\right| \\
& +C_{3} \int_{0}^{t} D_{s}(m, \tilde{m}) d s
\end{aligned}
$$

which together with Gronwall's lemma gives

$$
\sup _{0 \leq s \leq t}|x(s)-\tilde{x}(s)| \wedge 1 \leq C_{2} t \sup _{a \in \mathcal{A},(t, x) \in \bar{Q}_{T}}\left|\phi_{a}-\tilde{\phi}_{a}\right|+C_{3} \int_{0}^{t} D_{s}(m, \tilde{m}) d s .
$$

Subsequently,

$$
D_{t}(m, \tilde{m}) \leq C_{2} t \sup _{a \in \mathcal{A},(t, x) \in \bar{Q}_{T}}\left|\phi_{a}-\tilde{\phi}_{a}\right|+C_{3} \int_{0}^{t} D_{s}(m, \tilde{m}) d s .
$$


By using Gronwall's lemma again, we complete the proof.

TheOrem 10. Assume (H1)-(H6) hold. If the constants $c_{1}$ for (37) and $c_{2}$ for (38) can be selected to satisfy the composite gain $(C G)$ condition $c_{1} c_{2}<1$, then there exists a unique solution for (35) and hence a unique solution for the NCE equation system (9)-(11).

Proof. It follows easily from a fixed point argument that there is a unique $m \in$ $\mathcal{M}\left(C^{(K)}\right)$ satisfying equation (36). Consequently, we can construct $\mu_{\text {. }}^{o}=p_{\mu}(m)$ to satisfy (35). Assume we have two measure flows $\mu_{.}^{o}, \tilde{\mu}^{o} . \in \mathcal{M}_{[0, T]}$ satisfying (35), and then by the construction of the mappings $\Gamma, \bar{\Gamma}, \widehat{\Gamma}$, it is easy to derive the associated fixed points $m$ and $\tilde{m}$ for (36) such that $\mu^{o}=p_{\mu}(m)$ and $\tilde{\mu}^{o}=p_{\mu}(\tilde{m})$. Since we necessarily have $m=\tilde{m}$, it follows that $\mu_{\text {. }}^{o}=\tilde{\mu}_{\text {. }}^{o}$. This completes the proof.

7.1. Discussion on Feedback Regularity. For illustrating the inequality in the FR condition (37), we examine a highly simplified situation by considering a linear quadratic model with nonlinear coupling, which is a variant of (2). In addition, there is no bound constraints on the control. We note that for general nonlinear models, it is much more difficult to obtain the corresponding estimates explicitly.

We consider a system of uniform agents, i.e., $\mathcal{A}$ degenerates to a singleton. Let the dynamics be given by $d z_{i}=a z_{i} d t+b u_{i} d t+(\alpha / n) \sum_{j=1}^{n} \phi\left(z_{j}\right) d t+\sigma d w_{i}$, and the cost is $J_{i}=E \int_{0}^{T}\left[\left(z_{i}-(\beta / n) \sum_{j=1}^{n} \psi\left(z_{j}\right)\right)^{2}+r u_{i}^{2}\right] d t, 1 \leq i \leq n$. First, assume two measure flows $\mu_{t} \triangleq \mu_{t}^{(1)}$ and $\tilde{\mu}_{t} \triangleq \mu_{t}^{(2)}$ on $[0, T]$, which are the marginal distributions on $\mathbb{R}$, respectively, of two probability measures $m_{1}$ and $m_{2}$, both in $\mathcal{M}\left(C^{(1)}\right)$. We determine two sets of functions $f^{(k)}(t)=\int_{\mathbb{R}} \phi(y) \mu_{t}^{(k)}(d y), z^{*(k)}(t)=\int_{\mathbb{R}} \psi(y) \mu_{t}^{(k)}(d y)$, where $k=1,2, t \in[0, T]$ and $\phi, \psi$ are bounded and Lipschitz continuous functions on $\mathbb{R}$. Accordingly, we obtain two sets of control laws

$$
u_{t}^{(k)}=\varphi^{(k)}\left(t, z_{i}\right)=-\frac{b}{r}\left(\Pi_{t} z_{i}+s_{t}^{(k)}\right), \quad k=1,2,
$$

which minimizes $J_{i}=E \int_{0}^{T}\left[\left(z_{i}-\beta z^{*(k)}\right)^{2}+r u_{i}^{2}\right] d t$ subject to $d z_{i}=a z_{i} d t+b u_{i} d t+$ $\alpha f^{(k)} d t+\sigma d w_{i}$. In the above, we have

$$
\begin{aligned}
& \frac{d \Pi_{t}}{d t}+2 a \Pi_{t}-\frac{b^{2}}{r} \Pi_{t}^{2}+1=0, \\
& \frac{d s_{t}^{(k)}}{d t}+\left(a-\frac{b^{2}}{r} \Pi_{t}\right) s_{t}^{(k)}+\alpha \Pi_{t} f^{(k)}-\beta z^{*(k)}=0,
\end{aligned}
$$

where $0 \leq t \leq T$, and $\Pi_{T}=0, s_{T}^{(k)}=0$. Let $C_{\Pi}=\sup _{0 \leq t \leq T} \Pi_{t}$. For a proof of the optimality of the control law, see e.g. [9]. By combining Gronwall's lemma with (41), we see that there exist constants $c_{1}$ and $c_{2}$ depending upon $C_{\Pi}$ such that

$$
\sup _{0 \leq t \leq T}\left|s_{t}^{(2)}-s_{t}^{(1)}\right| \leq c_{1}|\alpha| \sup _{0 \leq \tau \leq T}\left|f^{(2)}(\tau)-f^{(1)}(\tau)\right|+c_{2}|\beta| \sup _{0 \leq \tau \leq T}\left|z^{*(2)}(\tau)-z^{*(1)}(\tau)\right| .
$$


Letting $g$ stand for $\phi$ and $\psi$, we have

$$
\begin{aligned}
\int g(x) \mu_{t}(d x)-\int g(x) \tilde{\mu}_{t}(d x) & =\int g\left(X_{t}\left(\omega_{1}\right)\right) d m_{1}\left(\omega_{1}\right)-\int g\left(X_{t}\left(\omega_{2}\right)\right) d m_{2}\left(\omega_{2}\right) \\
& =\int g\left(X_{t}\left(\omega_{1}\right)\right) d m\left(\omega_{1}, \omega_{2}\right)-\int g\left(X_{t}\left(\omega_{2}\right)\right) d m\left(\omega_{1}, \omega_{2}\right)
\end{aligned}
$$

where $X$ is the canonical process and $m$ is a coupling of $m_{1}$ and $m_{2}$. Hence

$$
\begin{aligned}
& \left|\int g(x) \mu_{t}(d x)-\int g(x) \tilde{\mu}_{t}(d x)\right| \\
\leq & c_{3} \int_{C\left([0, T], \mathbb{R}^{2}\right)} \sup _{0 \leq t \leq T}\left(\left|X_{t}\left(\omega_{1}\right)-X_{t}\left(\omega_{2}\right)\right| \wedge 1\right) d m\left(\omega_{1}, \omega_{2}\right)
\end{aligned}
$$

for a constant $c_{3}$ depending upon $\phi$ and $\psi$. Noticing that $\left|\varphi^{(2)}(t, z)-\varphi^{(1)}(t, z)\right|=$ $\frac{|b|}{r}\left|s_{t}^{(2)}-s_{t}^{(1)}\right|$, we get

$$
\left|\varphi^{(2)}(t, z)-\varphi^{(1)}(t, z)\right| \leq \frac{|b|}{r} c_{3}\left(c_{1}|\alpha|+c_{2}|\beta|\right) D_{T}\left(m_{1}, m_{2}\right) .
$$

It is seen that the coefficient $\frac{|b|}{r} c_{3}\left(c_{1}|\alpha|+c_{2}|\beta|\right)$ can be made sufficiently small by choosing suitably small $\alpha$ and $\beta$.

8. Asymptotic Equilibrium Analysis. Within the context of a population of $n$ agents with dynamics (1), for any $1 \leq k \leq n$, the $k$ th agent's admissible control set $\mathcal{U}_{k}$ consists of all Lipschitz feedback controls $u_{k}$ adapted to the $\sigma$-algebra $\mathcal{F}\left(z_{i}(\tau), \tau \leq\right.$ $t, 1 \leq i \leq n$ ) (i.e., $u_{k}(t)$ is a continuous function of $\left(t, z_{1}(t), \cdots, z_{n}(t)\right)$ and Lipschitz continuous in $\left.\left(z_{1}(t), \cdots, z_{n}(t)\right)\right)$, which ensures a unique strong solution to the closedloop system of the $n$ agents exists on $[0, \infty)$. In parallel, we define $\mathcal{U}_{k}^{d}$, as a subset of $\mathcal{U}_{k}$, such that the Lipschitz feedback control $u_{k}(t)$ depends upon $\left(t, z_{k}(t)\right)$. We denote $\mathcal{U}^{n}=\mathcal{U}_{1} \times \cdots \times \mathcal{U}_{n}$ and $\mathcal{U}^{d, n}=\mathcal{U}_{1}^{d} \times \cdots \times \mathcal{U}_{n}^{d}$.

Note that $\mathcal{U}_{k}$ is not restricted to be decentralized, and this will give a stronger characterization of the $\varepsilon$-Nash strategies introduced below. For each $i$, we denote by $u_{-i}$ the vector obtained by deleting $u_{i}$ in $\left(u_{1}, \cdots, u_{i}, \cdots, u_{n}\right)$.

For the dynamic game problem specified by (1)-(2), we rewrite the individual cost (2) for the $i$ th agent in the form

$$
J_{i}\left(u_{i}, u_{-i}\right)=E \int_{0}^{T}\left[(1 / n) \sum_{j=1}^{n} L\left(z_{i}, u_{i}, z_{j}\right)\right] d t, \quad 1 \leq i \leq n,
$$

which indicates the effect of the control laws of other agents due to the coupling in dynamics and individual costs.

Definition 11. For the $n$ players, let the costs be given by $J_{k}, 1 \leq k \leq n$. A set of controls $u_{k} \in \mathcal{U}_{k}$ (resp., $u_{k} \in \mathcal{U}_{k}^{d}$ ) each given as a Lipschitz feedback $\varphi_{k}, 1 \leq k \leq n$, is called an $\varepsilon$-Nash equilibrium with respect to the strategy space $\mathcal{U}^{n}$ (resp., $\mathcal{U}^{d, n}$ ), if 
there exists $\varepsilon \geq 0$ such that for any fixed $1 \leq i \leq n$, we have

$$
J_{i}\left(u_{i}, u_{-i}\right) \leq J_{i}\left(u_{i}^{\prime}, u_{-i}\right)+\varepsilon,
$$

when any alternative $u_{i}^{\prime} \in \mathcal{U}_{i}$ (resp., $u_{i}^{\prime} \in \mathcal{U}_{i}^{d}$,), determined as another Lipschitz feedback $\tilde{\varphi}_{i}$, is applied by the ith player.

In relation to $\left(u_{i}, u_{-i}\right)$ in Definition 11 , we may also call $u_{i}$ an $\varepsilon$-Nash strategy. This is with an underlying assumption that other players use strategies $u_{-i}$.

8.1. Decoupling Rate and Crossing Perturbation. Let $\phi_{\theta_{k}}(t, x) \in C_{L i p(x)}$ $\left(\bar{Q}_{T}, U\right), 1 \leq k \leq K$, be a set of $K$ functions associated with different values $\theta_{k}$, not necessarily satisfying the NCE principle. These functions are used by the $n$ agents for their individual control laws. We write the closed-loop equation as follows:

$$
d z_{i}=(1 / n) \sum_{j=1}^{n} f_{a_{i}}\left(z_{i}, \phi_{a_{i}}\left(t, z_{i}\right), z_{j}\right) d t+\sigma d w_{i}, \quad 1 \leq i \leq n,
$$

where $\phi_{a_{i}}$ reduces to $\phi_{\theta_{k}}$ if $a_{i}=\theta_{k}$. By the Lipschitz condition of the feedback, we see that there exists a unique strong solution $\left(z_{1}, \cdots, z_{n}\right)$ on $[0, T]$. For the agent with dynamic parameter $a_{i}$, we write the associated M-V equation system as follows

$$
d \hat{z}_{i}=f_{a_{i}}\left[\hat{z}_{i}, \phi_{a_{i}}\left(t, \hat{z}_{i}\right), \mu_{t}^{1}, \cdots, \mu_{t}^{K}\right] d t+\sigma d w_{i}, \quad 1 \leq i \leq n,
$$

where the initial condition is $\hat{z}_{i}(0)=z_{i}(0)$. In contrast to the coupled processes in (43), equation (44) gives $n$ independent processes. Denote

$$
\varepsilon_{n}=\sup _{1 \leq k \leq K}\left|F_{n}\left(\left\{\theta_{k}\right\}\right)-\pi_{k}\right|
$$

which measures the gap between the empirical distribution of the dynamic parameter and its limit. Under (H0), we have $\lim _{n \rightarrow \infty} \varepsilon_{n}=0$.

We have the following decoupling result which shows that each process $z_{i}$ may be approximated by the corresponding process $\hat{z}_{i}$ as $n \rightarrow \infty$.

TheOREM 12. Assume (HO)-(H3) and let $z_{i}$ and $\hat{z}_{i}, 1 \leq i \leq n$, be given by (43)-(44). We have

$$
\sup _{1 \leq i \leq n} E \sup _{0 \leq t \leq T}\left|z_{i}(t)-\hat{z}_{i}(t)\right|^{\kappa}=O\left(n^{-1 / 2}+\varepsilon_{n}\right)
$$

where $\kappa=1,2, \varepsilon_{n}$ is given by (45), and the right hand side may depend upon the terminal time $T$.

Proof. We begin by considering the case $\kappa=1$. Denote the relation $j \in S\left(\theta_{k}\right)$ if 
the dynamic parameter associated with $z_{j}$ is $a_{j}=\theta_{k}$. It follows that

$$
\begin{aligned}
z_{i}(s)-\hat{z}_{i}(s)=\int_{0}^{s} & (1 / n) \sum_{j=1}^{n} f_{a_{i}}\left(z_{i}, \phi_{a_{i}}\left(t, z_{i}\right), z_{j}\right) d t \\
& \quad-\int_{0}^{s} f_{a_{i}}\left[\hat{z}_{i}, \phi_{a_{i}}\left(t, \hat{z}_{i}\right), \mu_{t}^{1}, \cdots, \mu_{t}^{K}\right] d t \\
= & \int_{0}^{s} \sum_{k=1}^{K} \sum_{j \in S\left(\theta_{k}\right)}(1 / n) f_{a_{i}}\left(z_{i}, \phi_{a_{i}}\left(t, z_{i}\right), z_{j}\right) d t \\
& \quad-\int_{0}^{s} \sum_{k=1}^{K} \pi_{k} \int_{y \in \mathbb{R}} f_{a_{i}}\left(\hat{z}_{i}, \phi_{a_{i}}\left(t, \hat{z}_{i}\right), y\right) \mu_{t}^{k}(d y) d t .
\end{aligned}
$$

Let

$$
D_{k}^{i}(t) \triangleq \sum_{j \in S\left(\theta_{k}\right)}(1 / n) f_{a_{i}}\left(z_{i}, \phi_{a_{i}}\left(t, z_{i}\right), z_{j}\right)-\pi_{k} \int_{\mathbb{R}} f_{a_{i}}\left(\hat{z}_{i}, \phi_{a_{i}}\left(t, \hat{z}_{i}\right), y\right) \mu_{t}^{k}(d y) .
$$

We have

$$
\begin{aligned}
& D_{k}^{i}(t) \\
= & \sum_{j \in S\left(\theta_{k}\right)}(1 / n) f_{a_{i}}\left(z_{i}, \phi_{a_{i}}\left(t, z_{i}\right), z_{j}\right)-\sum_{j \in S\left(\theta_{k}\right)}(1 / n) f_{a_{i}}\left(\hat{z}_{i}, \phi_{a_{i}}\left(t, \hat{z}_{i}\right), z_{j}\right) \quad\left(\triangleq D_{k, 1}^{i}\right) \\
& +\sum_{j \in S\left(\theta_{k}\right)}(1 / n) f_{a_{i}}\left(\hat{z}_{i}, \phi_{a_{i}}\left(t, \hat{z}_{i}\right), z_{j}\right)-\sum_{j \in S\left(\theta_{k}\right)}(1 / n) f_{a_{i}}\left(\hat{z}_{i}, \phi_{a_{i}}\left(t, \hat{z}_{i}\right), \hat{z}_{j}\right) \quad\left(\triangleq D_{k, 2}^{i}\right) \\
& +\sum_{j \in S\left(\theta_{k}\right)}(1 / n) f_{a_{i}}\left(\hat{z}_{i}, \phi_{a_{i}}\left(t, \hat{z}_{i}\right), \hat{z}_{j}\right)-\pi_{k} \int f_{a_{i}}\left(\hat{z}_{i}, \phi_{a_{i}}\left(t, \hat{z}_{i}\right), y\right) \mu_{t}^{k}(d y) . \quad\left(\triangleq D_{k, 3}^{i}\right)
\end{aligned}
$$

By the Lipschitz continuity condition of $\phi_{a_{i}}$ and $f_{a_{i}}$, there exists a constant $C>0$, independent of $n$, such that

$$
\left|D_{k, 1}^{i}\right|+\left|D_{k, 2}^{i}\right| \leq C \sum_{j \in S\left(\theta_{k}\right)}(1 / n)\left[\left|z_{i}-\hat{z}_{i}\right|+\left|z_{j}-\hat{z}_{j}\right|\right] .
$$

It follows from (46)-(47) that

$$
\begin{aligned}
\sup _{0 \leq s \leq t}\left|z_{i}(s)-\hat{z}_{i}(s)\right| \leq & C \int_{0}^{t}\left|z_{i}(s)-\hat{z}_{i}(s)\right| d s+C \int_{0}^{t}(1 / n) \sum_{j=1}^{n}\left|z_{j}(s)-\hat{z}_{j}(s)\right| d s \\
& +\int_{0}^{t} \sum_{k=1}^{K}\left|D_{k, 3}^{i}(s)\right| d s,
\end{aligned}
$$

which gives

$$
\begin{aligned}
\sum_{i=1}^{n} \sup _{0 \leq s \leq t}\left|z_{i}(s)-\hat{z}_{i}(s)\right| & \leq 2 C \sum_{i=1}^{n} \int_{0}^{t}\left|z_{i}(s)-\hat{z}_{i}(s)\right| d s+\int_{0}^{t} \sum_{i=1}^{n} \sum_{k=1}^{K}\left|D_{k, 3}^{i}(s)\right| d s \\
& \leq 2 C \sum_{i=1}^{n} \int_{0}^{t} \sup _{0 \leq \tau \leq s}\left|z_{i}(\tau)-\hat{z}_{i}(\tau)\right| d s+\int_{0}^{t} \sum_{i=1}^{n} \sum_{k=1}^{K}\left|D_{k, 3}^{i}(s)\right| d s .
\end{aligned}
$$


Now we show

$$
\sup _{0 \leq t \leq T} \sum_{k=1}^{K} E\left|D_{k, 3}^{i}(t)\right|=O\left(n^{-1 / 2}+\varepsilon_{n}\right)
$$

In fact, we have

$$
\begin{aligned}
& E\left|D_{k, 3}^{i}(t)\right|^{2} \\
& \leq 2 E\left|\sum_{j \in S\left(\theta_{k}\right)}\left[(1 / n) f_{a_{i}}\left(\hat{z}_{i}, \phi_{a_{i}}\left(t, \hat{z}_{i}\right), \hat{z}_{j}\right)-\int(1 / n) f_{a_{i}}\left(\hat{z}_{i}, \phi_{a_{i}}\left(t, \hat{z}_{i}\right), y\right) \mu_{t}^{k}(d y)\right]\right|^{2} \\
& \quad+2 E\left|\left[(1 / n) \sum_{j=1}^{n} 1_{\left\{j \in S\left(\theta_{k}\right)\right\}}-\pi_{k}\right] \int f_{a_{i}}\left(\hat{z}_{i}, \phi_{a_{i}}\left(t, \hat{z}_{i}\right), y\right) \mu_{t}^{k}(d y)\right|^{2} \\
& \triangleq 2 S_{1}+2 S_{2} .
\end{aligned}
$$

We clearly have $S_{2}=O\left(\varepsilon_{n}^{2}\right)$ by (H0). By independence of the components $\hat{z}_{j}, 1 \leq$ $j \leq n$, the crossing terms in $S_{1}$ generated by taking $j^{\prime} \neq j^{\prime \prime}$ (for $j$ ) is zero after taking expectations, which implies $S_{1}=O(1 / n)$. Thus it follows that

$$
E\left|D_{k, 3}^{i}(t)\right|^{2}=O\left(1 / n+\varepsilon_{n}^{2}\right),
$$

where the right hand side does not depend upon $t$, and therefore, $E\left(\sum_{k=1}^{K}\left|D_{k, 3}^{i}(t)\right|\right)^{2}$ $=O\left(1 / n+\varepsilon_{n}^{2}\right)$ by the elementary inequality $\left(y_{1}+\cdots+y_{K}\right)^{2} \leq K\left(y_{1}^{2}+\cdots+y_{K}^{2}\right)$ for real numbers. Hence $E \sum_{k=1}^{K}\left|D_{k, 3}^{i}(s)\right|=O\left(n^{-1 / 2}+\varepsilon_{n}\right)$, and (50) follows.

Now by (50) and (49), it follows from Gronwall's lemma that

$$
\sum_{i=1}^{n} E \sup _{0 \leq s \leq T}\left|z_{i}(s)-\hat{z}_{i}(s)\right|=O\left(n^{1 / 2}+n \varepsilon_{n}\right),
$$

which, combined again with Gronwall's lemma and (48), yields $E \sup _{0 \leq s \leq T} \mid z_{i}(s)-$ $\hat{z}_{i}(s) \mid=O\left(n^{-1 / 2}+\varepsilon_{n}\right)$. This completes the case for $\kappa=1$.

By combining the finished part with boundedness of $\sup _{1 \leq i \leq n} \sup _{0 \leq t \leq T} \mid z_{i}(t)-$ $\hat{z}_{i}(t) \mid$, the case with $\kappa=2$ follows. This completes the proof.

The following performance analysis involves extensive crossing perturbation estimate. Specifically, when a given agent changes its control, it will result in state process variations for other agents. In turn, these variations, together with the initial control change, will affect the dynamics of that agent.

TheOREm 13. Assume (HO)-(H6) hold and there exists a set of Lipschitz control laws $\left(u_{1}, \cdots, u_{n}\right)=\left(\varphi_{a_{1}}, \cdots, \varphi_{a_{n}}\right)$ satisfying the NCE principle, i.e., the set of control laws $\left(\varphi_{\theta_{1}}, \cdots, \varphi_{\theta_{K}}\right)$ is derived from the HJB equation (9) together with the $M-V$ equation (11), and satisfies the Lipschitz condition (H6). When the ith agent changes its control from $u_{i}$ to $u_{i}^{\prime} \in \mathcal{U}_{i}^{d}$ and the control laws of all other agents remain the same, the cost $J_{i}\left(u_{i}^{\prime}, u_{-i}\right)$ can be decreased by at most $O\left(n^{-1 / 2}+\varepsilon_{n}\right)$, i.e., $J_{i}\left(u_{i}^{\prime}, u_{-i}\right) \geq J_{i}\left(u_{i}, u_{-i}\right)-O\left(n^{-1 / 2}+\varepsilon_{n}\right)$, where $\varepsilon_{n}$ is given by $(45)$. 
Proof. To simplify the notation, we consider a strategy change for the first agent. We write

$$
d z_{i}=(1 / n) \sum_{j=1}^{n} f_{a_{1}}\left(z_{i}, \varphi_{a_{i}}\left(t, z_{i}\right), z_{j}\right) d t+\sigma d w_{i}, \quad 1 \leq i \leq n
$$

and

$$
\left\{\begin{aligned}
d z_{1}^{\prime} & =(1 / n) \sum_{j=1}^{n} f_{a_{1}}\left(z_{1}^{\prime}, \tilde{\varphi}_{a_{1}}\left(t, z_{1}^{\prime}\right), z_{j}^{\prime}\right) d t+\sigma d w_{1} \\
d z_{2}^{\prime} & =(1 / n) \sum_{j=1}^{n} f_{a_{2}}\left(z_{2}^{\prime}, \varphi_{a_{2}}\left(t, z_{2}^{\prime}\right), z_{j}^{\prime}\right) d t+\sigma d w_{2} \\
& \vdots \\
d z_{n}^{\prime} & =(1 / n) \sum_{j=1}^{n} f_{a_{n}}\left(z_{n}^{\prime}, \varphi_{a_{n}}\left(t, z_{n}^{\prime}\right), z_{j}^{\prime}\right) d t+\sigma d w_{n}
\end{aligned}\right.
$$

where the equation system (52) gives the closed-loop dynamics after the first agent takes the control $\tilde{\varphi}_{a_{1}}$ instead of $\varphi_{a_{1}}$. The two equation systems (51) and (52) have the same initial condition $\left(z_{1}(0), \cdots, z_{n}(0)\right)$. We further introduce the auxiliary M-V equation system

$$
d z_{i}^{\prime \prime}=f_{a_{i}}\left[z_{i}^{\prime \prime}, \varphi_{a_{i}}\left(t, z_{i}^{\prime \prime}\right), \mu_{t}^{1}, \cdots, \mu_{t}^{K}\right] d t+\sigma d w_{i},
$$

where the initial condition is still $\left(z_{1}(0), \cdots, z_{n}(0)\right)$.

We compare $\left(z_{2}, \cdots, z_{n}\right)$ and $\left(z_{2}^{\prime}, \cdots, z_{n}^{\prime}\right)$ in (51) and (52) by treating $z_{1}$ and $z_{1}^{\prime}$ as an additional quantity, and show by Gronwall's lemma that

$$
\sup _{2 \leq j \leq n} \sup _{0 \leq s \leq T}\left|z_{j}(s)-z_{j}^{\prime}(s)\right| \leq O(1 / n),
$$

where the right hand side holds uniformly with respect to the choices of $\tilde{\varphi}_{a_{1}}$.

By the same argument as in proving Theorem 12, we get

$$
\sup _{2 \leq j \leq n} E \sup _{0 \leq t \leq T}\left|z_{j}^{\prime \prime}(t)-z_{j}^{\prime}(t)\right|^{\kappa}+\sup _{1 \leq j \leq n} E \sup _{0 \leq t \leq T}\left|z_{j}^{\prime \prime}(t)-z_{j}(t)\right|^{\kappa}=O\left(n^{-1 / 2}+\varepsilon_{n}\right),
$$

for $\kappa=1,2$.

We introduce the new equation

$$
d \hat{z}_{1}^{\prime}=(1 / n) \sum_{j=1}^{n} f_{a_{1}}\left(\hat{z}_{1}^{\prime}, \tilde{\varphi}_{a_{1}}\left(t, \hat{z}_{1}^{\prime}\right), z_{j}^{\prime \prime}\right) d t+\sigma d w_{1}
$$

where $\hat{z}_{1}^{\prime}(0)=z_{1}(0)$ and $\left(z_{1}^{\prime \prime}, \cdots, z_{n}^{\prime \prime}\right)$ is given by (53). By (52) and (55), once $\tilde{\varphi}_{a_{1}}$ is selected, we can show that

$$
E \sup _{0 \leq t \leq T}\left|\hat{z}_{1}^{\prime}(t)-z_{1}^{\prime}(t)\right|^{\kappa}=O\left(n^{-1 / 2}+\varepsilon_{n}\right), \quad \kappa=1,2 .
$$

Next, we construct

$$
d \tilde{z}_{1}^{\prime}=f_{a_{1}}\left[\tilde{z}_{1}^{\prime}, \tilde{\varphi}_{a_{1}}\left(t, \tilde{z}_{1}^{\prime}\right), \mu_{t}^{1}, \cdots, \mu_{t}^{K}\right] d t+\sigma d w_{1},
$$


where $\tilde{z}_{1}^{\prime}(0)=z_{1}(0)$. Combining (55)-(56) and applying a similar argument as in proving the decoupling results in Theorem 12, we can show that

$$
E \sup _{0 \leq t \leq T}\left|\hat{z}_{1}^{\prime}-\tilde{z}_{1}^{\prime}\right|=O\left(n^{-1 / 2}+\varepsilon_{n}\right) .
$$

Subsequently, we can show that

$$
\begin{aligned}
& E \int_{0}^{T}(1 / n) \sum_{j=1}^{n} L\left(z_{1}^{\prime}, \tilde{\varphi}_{a_{1}}\left(t, z_{1}^{\prime}\right), z_{j}^{\prime}\right) d t \\
& \geq E \int_{0}^{T}(1 / n) \sum_{j=1}^{n} L\left(z_{1}^{\prime}, \tilde{\varphi}_{a_{1}}\left(t, z_{1}^{\prime}\right), z_{j}^{\prime \prime}\right) d t-O\left(n^{-1 / 2}+\varepsilon_{n}\right) \\
& \geq E \int_{0}^{T}(1 / n) \sum_{j=1}^{n} L\left(\tilde{z}_{1}^{\prime}, \tilde{\varphi}_{a_{1}}\left(t, \tilde{z}_{1}^{\prime}\right), z_{j}^{\prime \prime}\right) d t-O\left(n^{-1 / 2}+\varepsilon_{n}\right) \\
& \geq E \int_{0}^{T} L\left[\tilde{z}_{1}^{\prime}, \tilde{\varphi}_{a_{1}}\left(t, \tilde{z}_{1}^{\prime}\right), \mu_{t}^{1}, \cdots, \mu_{t}^{K}\right] d t-O\left(n^{-1 / 2}+\varepsilon_{n}\right) \\
& \geq E \int_{0}^{T} L\left[z_{1}^{\prime \prime}, \varphi_{a_{1}}\left(t, z_{1}^{\prime \prime}\right), \mu_{t}^{1}, \cdots, \mu_{t}^{K}\right] d t-O\left(n^{-1 / 2}+\varepsilon_{n}\right),
\end{aligned}
$$

where the last inequality follows from the optimality interpretation of $\varphi_{a_{1}}$ for (53). By further comparing $E \int_{0}^{T} L\left[z_{1}^{\prime \prime}, \varphi_{a_{1}}\left(t, z_{1}^{\prime \prime}\right), \mu_{t}^{1}, \cdots, \mu_{t}^{K}\right] d t$ with the cost $E \int_{0}^{T}(1 / n)$ $\sum_{j=1}^{n} L\left(z_{1}, \varphi_{a_{1}}\left(t, z_{1}\right), z_{j}\right) d t$ under the control laws $\left(\varphi_{a_{1}}, \cdots, \varphi_{a_{n}}\right)$ in (51) for the $n$ agents, we can show the theorem holds when $u_{1}$ changes within $\mathcal{U}_{1}^{d}$. This completes the proof.

In Theorem 13, the equilibrium analysis is based upon the general nonlinear dynamics and we need to restrict the strategy change within the set $\mathcal{U}_{i}^{d}$. This simplifies the performance comparison. Now we proceed to consider the strategy change in the wider set $\mathcal{U}_{i}$. To get a tractable analysis, meanwhile, we need to introduce more structural information into the system model. In particular, we consider individual dynamics and costs involving both additive and multiplicative coupling. The corollary below may be proved by following the above argument in establishing Theorem 13 and we will not repeat the details here.

Corollary 14. Assume the conditions in Theorem 13 hold, the individual dynamics may be decomposed into the form

$$
f_{a_{i}}\left(z_{i}, u_{i}, z_{j}\right)=f_{a_{i}}^{0}\left(z_{i}, u_{i}\right) g_{a_{i}}^{0}\left(z_{j}\right)+g_{a_{i}}^{1}\left(z_{j}\right),
$$

and the cost is given in the form $J_{i}\left(u_{i}, u_{-i}\right)=E \int_{0}^{T}\left\{(1 / n) \sum_{j=1}^{n}\left[L^{0}\left(z_{i}, u_{i}\right) h^{0}\left(z_{j}\right)+\right.\right.$ $\left.\left.h^{1}\left(z_{j}\right)\right]\right\} d t, 1 \leq i \leq n$. Also, assume the individual controls $\left(u_{1}, \cdots, u_{n}\right)=\left(\varphi_{a_{1}}, \cdots\right.$, $\left.\varphi_{a_{n}}\right)$ are specified by the NCE principle as in Theorem 13. Then $\left(u_{1}, \cdots, u_{n}\right)$ is an $\varepsilon$-Nash strategy with respect to $\mathcal{U}^{n}$, where $\varepsilon \rightarrow 0$ as $n \rightarrow \infty$.

We note that the result in Corollary 14 may be further extended to models with a cost integrand of the form $\Theta\left(z_{i}, u_{i},(1 / n) \sum_{j=1}^{n} h\left(z_{j}\right)\right)$ for $J_{i}$. Hence, our NCE 
methodology can deal with many typical models with mean field coupling. It would be of interest to consider the asymptotic equilibrium analysis with respect to the strategy space $\mathcal{U}^{n}$ for more general nonlinear models than that appearing in Corollary 14 . This will depend upon developing more sophisticated crossing perturbation analysis and will be investigated in future work.

\section{REFERENCES}

[1] R. J. Aumann, Values of markets with a continuum of traders. Econometrica, 43(1975), pp. 611-646.

[2] R. J. Aumann and L. S. Shapley, Values of Non-Atomic Games. Princeton Univ. Press, Princeton, NJ, 1974.

[3] F. Baccelli, D. Hong, And Z. Liu, Fixed point methods for the simulation of the sharing of a local loop by a large number of interacting TCP connections. INRIA Tech. Rep. No. 4154, France, 2001.

[4] E. J. BALDER, An equilibrium existence result for games with incomplete information and indeterminate outcomes. J. Math. Econ., 40:3(2004), pp. 297-320.

[5] R. Balescue, Equilibrium and Nonequilibrium Statistical Mechanics. John Wiley, New York, 1975.

[6] T. Başar And G. J. Olsder, Dynamic Noncooperative Game Theory, 2nd ed. Academic Press, London, UK, 1995.

[7] S. Benachour, B. Roynette, D. Talay, and P. Vallois, Nonlinear self-stabilizing processes. I: existence, invariant probability, propagation of chaos. Stochastic Processes Appl., vol. 75, no. 2, pp. 173-201, 1998.

[8] S. Benachour, B. Roynette, and P. Vallois, Nonlinear self-stabilizing processes. II: convergence to invariant probability. Stochastic Processes Appl., vol. 75, no. 2, pp. 203-224, 1998.

[9] A. Bensoussan, Stochastic Control of Partially Observable Systems. Cambridge Univ. Press, 1992.

[10] D. Chandler, Introduction to Modern Statistical Mechanics. Oxford University Press, New York, 1987.

[11] D. A. Dawson, Critical dynamics and fluctuations for a mean-field model of cooperative behavior. J. Statist. Phys., 31:1(1983), pp. 29-85.

[12] D. A. Dawson And J. GäRTner, Large deviations from the McKean-Vlasov limit for weakly interacting diffusions. Stochastics, 20(1987), pp. 247-308.

[13] R. L. Dobrushin, Prescribing a system of random variables by conditional distributions. Theory of Probab. and its Appl., 3(1970), pp. 458-486.

[14] G. M. ErICKson, Differential game models of advertising competition. Europ. J. Oper. Res., 83(1995), pp. 431-438.

[15] W. H. Fleming and R. W. Rishel, Deterministic and Stochastic Optimal Control, SpringerVerlag, New York, 1975.

[16] A. Friedman, Differential Games, Wiley-Interscience, 1971.

[17] J. W. Friedman, Game Theory with Applications to Economics, 2nd ed. Oxford University Press, New York, 1990.

[18] D. Fudenberg and D. K. Levine, The Theory of Learning in Games. MIT Press, Cambridge, MA, 1998.

[19] E. J. Green, Continuum and finite-player noncooperative models of competition. Economet- 
rica, 52:4(1984), pp. 975-993.

[20] J. C. Harsanyi, Games with incomplete information played by "Bayesian" players, Parts I, II, and III. Management Science, 14(1967-68), pp. 159-182, pp. 320-334, pp. 486-502.

[21] J. C. Harsanyi, Games with incomplete information. Amer. Econ. Rev., 85:3(1995), pp. 291303.

[22] A. Haurie And P. Marcotte, On the relationship between Nash-Cournot and Wardrop equilibria. Networks, 15(1985), pp. 295-308.

[23] M. Huang, P. E. Caines, and R. P. Malhamé, Individual and mass behaviour in large population stochastic wireless power control problems: centralized and Nash equilibrium solutions. Proc. 42nd IEEE Conf. Decision Contr., Maui, Hawaii, pp. 98-103, Dec. 2003.

[24] M. Huang, P. E. Caines, And R. P. Malhamé, Uplink power adjustment in wireless communication systems: a stochastic control analysis. IEEE Trans. Automat. Contr., 49(2004), pp. 1693-1708.

[25] M. Huang, P. E. Caines, And R. P. Malhamé, Large-population cost-coupled LQG problems: generalizations to non-uniform individuals. Proc. the 43rd IEEE Conference on Decision and Control, Paradise Island, Bahamas, pp. 3453-3458, December 2004.

[26] M. Huang, P. E. Caines, and R. P. Malhamé, Distributed multi-agent decision-making with partial observations: asymptotic Nash equilibria. Proc. the 17th Internat. Symposium on Math. Theory on Networks and Systems (MTNS'06), Kyoto, Japan, pp. 2725-2730, July 2006.

[27] M. Huang, P.E. Caines, and R.P. Malhamé, Large-population cost-coupled LQG problems with non-uniform agents: individual-mass behaviour and decentralized $\varepsilon$-Nash equilibria. IEEE Transactions on Automatic Control, 2007 (to appear).

[28] M. Huang, R. P. Malhamé, and P. E. Caines, Nash equilibria for large-population linear stochastic systems of weakly coupled agents. In: Analysis, Control and Optimization of Complex Dynamic Systems, E. K. Boukas and R. P. Malhamé Eds., Chapter 9, pp. 215252, Springer, New York, 2005.

[29] M. Huang, R. P. Malhamé, and P. E. Caines, Nash strategies and adaptation for decentralized games involving weakly-coupled agents. Proc. 44th IEEE CDC-ECC'05 Conf., Seville, Spain, pp. 1050-1055, Dec. 2005.

[30] M. Huang, R. P. Malhamé, and P. E. Caines, Nash certainty equivalence in large population stochastic dynamic games: connections with the physics of interacting particle systems. Proc. the 45th IEEE Conference on Decision and Control, San Diego, CA, pp. 4921-4926, Dec. 2006.

[31] R. P. IsAacs, Differential Games. John Wiley, 1965.

[32] M. KAC, Probability and Related Topics in Physical Science. Interscience, New York, 1958.

[33] M. Ali Khan and Y. Sun, Non-cooperative games with many players. In: Handbook of Game Theory with Economic Applications, Vol. 3, R. J. Aumann and S. Hart eds., North-Holland, 2002.

[34] Y. L. Klimontovich, Statistical Physics. Harwood Academic Publ., New York, 1986.

[35] V. E. Lambson, Self-enforcing collusion in large dynamic markets. J. Econ. Theory, 34(1984), pp. 282-291.

[36] J. Maynard Smith, Evolution and The Theory of Games. Cambridge Univ. Press, Cambridge, UK, 1982.

[37] H. P. McKean JR, A class of Markov processes associated with nonlinear parabolic equations. Proc. Nat. Acad. Sci., USA, 56(1966), pp. 1907-1911.

[38] J. M. McNamara, A. K. Houston, and E. J. Collins, Optimality models in behavioral biology. SIAM Review, 43:3(2001), pp. 413-466. 
[39] P. R. Milgrom and R. J. Weber, Distributional strategies for games with incomplete information. Math. Oper. Res., 10:4(1985), pp. 619-632.

[40] J. W. Milnor and L. S. Shapley, Values of large games II: Oceanic games. Math. Oper. Res., 3(1978), pp. 290-307.

[41] D. Morale, V. Capasso, and K. OelschläGer, An interacting particle system modelling aggregation behavior: from individual to populations. J. Math. Biol., 50(2005), pp. 49-66.

[42] G. P. Papavassilopoulos, On the linear-quadratic-Gaussian Nash game with one-step delay observation sharing pattern. IEEE Trans. Automat. Contr., 27:5(1982), pp. 1065-1071.

[43] N. Z. Shapiro and L. S. Shapley, Values of large games I: A limit theorem. Math. Oper. Res., 3(1978), pp. 1-9.

[44] A.-S. Sznitman, Topics in propagation of chaos. In: Ecole d'Eté de Probabilitiés de Saint-Flour XIX -1989, Lect. Notes Math. 1464, pp. 165-252, Springer-Verlag, Berlin, 1991.

[45] P. VaraiYa, N-player stochastic differential games. SIAM J. Contr. Optim., 14(1976), pp. 538-545.

[46] J. G. WARdrop, Some theoretical aspects of road traffic research. Proc. Inst. Civil Engineers, Part II, vol. 1, pp. 325-378, 1952. 